03,08

\title{
Электронная структура и пространственное распределение спиновой плотности мелких доноров азота в кристаллической решетке SiC
}

\author{
(С) М.В. Музафарова, И.В. Ильин, А.Н. Анисимов, Е.Н. Мохов, В.А. Солтамов, П.Г. Баранов \\ Физико-технический институт им. А.Ф. Иофрфе РАН, \\ Санкт-Петербург, Россия \\ ฯ E-mail: pavel.baranov@mail.ioffe.ru
}

(Поступила в Редакцию 24 мая 2016 г.)

\begin{abstract}
Открытие уникальных магнитооптических свойств спиновых центров в карбиде кремния, дающих возможность управлять спинами малых массивов центров атомарных размеров вплоть до одиночных центров при комнатных температурах, с использованием техники оптического детектирования магнитного резонанса, поставило ряд проблем, среди которых одной из основных является создание условий, при которых минимизируются эффекты спиновой релаксации. Как показали исследования свойств спиновых азотно-вакансионных центров в алмазе, основной вклад в спиновую релаксацию вносит взаимодействие с донорами азота, являющихся основной примесью в алмазе. В карбиде кремния существует подобная проблема, поскольку основной фоновой примесью также являются доноры азота. Задачей настоящей работы является исследование пространственного распределения спиновой плотности доноров азота в двух основных политипах карбида кремния $4 H-\mathrm{SiC}$ и $6 H-\mathrm{SiC}$, с целью использования этой информации для минимизации взаимодействия доноров азота со спиновыми центрами в карбиде кремния. Проанализированы результаты исследований методами магнитного резонанса и установлено распределение спиновой плотности на ближайших координационных сферах донора азота, занимающего положение углерода в карбиде кремния. Делается вывод, что спиновые центры в политипе $4 H-\mathrm{SiC}$, включающие вакансию кремния, могут быть более устойчивы к взаимодействиям с неспаренными донорными электронами, поскольку в этом случае электроны не локализуются на ближайшей к спиновому центру координационной сфере.
\end{abstract}

Работа была поддержана Министерством образования и науки РФ соглашение № 14.604.21.0083 (RFMEFI60414X0083).

\section{1. Введение}

Уникальные спиновые свойства азотно-вакансионных $(\mathrm{N} V)$-центров в алмазе открыли новую эру в спинтронике [1-4], которую даже назвали „алмазная эра в спинтронике“ (The diamond age of spintronics) [5]. Появилась возможность управлять спиновыми свойствами малых массивов центров атомарных размеров вплоть до одиночных центров в условиях окружающей среды, то есть при комнатных температурах, при этом использовалась техника оптического детектирования магнитного резонанса (ОДМР).

Последние десять лет ознаменовались открытием совершенно новых возможностей для спиновых центров в карбиде кремния ( $\mathrm{SiC})$, „Better than excellent" - это про карбид кремния в сравнении с алмазом [6]. Был запущен целый шквал исследований в этой области (см., например [7-30]). Было обнаружено по крайней мере два семейства однотипных центров, характеризующихся спинами $S=1$ и $S=3 / 2$, обладающих уникальными свойствами $\mathrm{N} V$-центров в алмазе, но существенно расширяющие их возможности. В этих центрах под действием оптической накачки происходит выстраивание населенностей спиновых подуровней как в основном, так и возбужденном спиновых состояниях. В результате применения резонансного микроволнового излучения или создания условий для антипересечения уровней (АПУ) спинового центра (level anticrossing - LAC) происходит резкое изменение интенсивности люминесценции даже при комнатных температурах. Таким образом, реализуется возможность управления спинами с помощью оптического и микроволнового возбуждения при комнатных температурах, что является весьма перспективным для применений в квантовых вычислениях и сенсорике с пространственным разрешением на микронном и субмикронном уровнях.

$\mathrm{SiC}$ является единственной химически устойчивой формой $\mathrm{Si}$ и С. Каждый атом $\mathrm{Si}(\mathrm{C})$ окружен четырьмя атомами C $(\mathrm{Si})$ в тетраэдрических $s p^{3}$-связях. Кристаллическая структура состоит из плотно упакованной последовательности слоев, содержащих атомы $\mathrm{Si}$ и C вдоль оси с. Изменение последовательности упаковки приводит к образованию различных политипов. Наиболее распространенными политипами являются $3 \mathrm{C}-\mathrm{SiC}$, имеющий кубическую симметрию, $4 H-$ - $6 H-\mathrm{SiC}$, имеющие гексагональную симметрию, и $15 R-\mathrm{SiC}$, имеющий ромбическую симметрию. В $4 H-\mathrm{SiC}$ реализуются две неэквивалентные кристаллографические позиции, гексагональная и квазикубическая позиция, названные $h$ и $k$ соответственно. В $6 H$-SiC реализуются три неэквивалентные позиции, одна гексагональная и две квазикубические, называемые $h, k 1$ и $k 2$. На рис. 1 показано схематическое представление кристаллической структуры $4 H$ - и $6 H$-SiC. Кристалл $\mathrm{SiC}$ состоит из тетраэдров, с четырьмя связями для каждого атома. Две из связей лежат в плоскости $\{11 \overline{2} 0\}$, ориентированные параллельно 


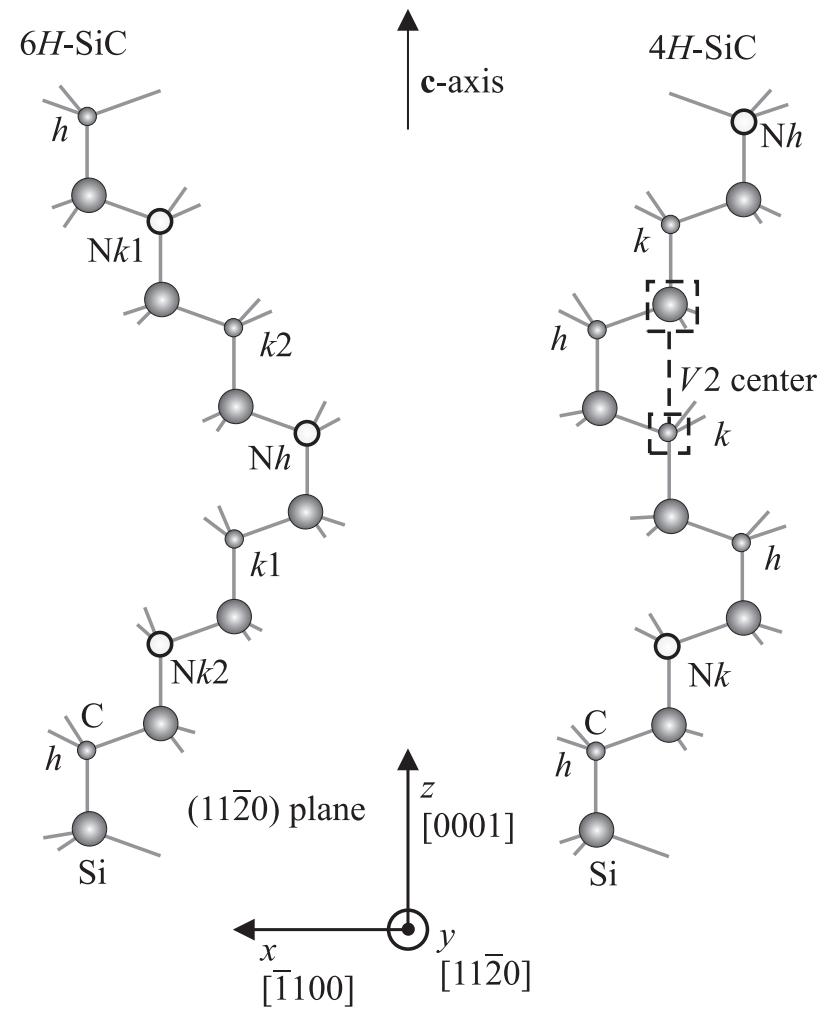

Рис. 1. Схематическое представление кристаллической структуры $4 H$ - и $6 H$-SiC. Кристалл $\mathrm{SiC}$ построен из тетраэдров, с четырьмя связями для каждого атома. Две из связей лежат в плоскости $(11 \overline{2} 0)$, совпадающей с плоскостью рисунка, одна из связей параллельна направлению оси с. Для $6 H-\mathrm{SiC}$ эта структура приводит к появлению трех неэквивалентных позиций $k 1, k 2$ и $h$, для $4 H$-SiC существуют только две позиции $k$ и $h$. Внизу - определение лабораторной системы координат, связанной с кристаллической симметрией, используемой в этой главе. Показаны возможные позиции донора азота; для $4 H$-SiC показана структура спинового центра $V 2$ со спином $S=3 / 2$.

и перпендикулярно направлению оси с. Различие между $h$ - и $k$-позициями возникает из-за разного положения атомов во второй координационной сфере. В $6 H-\mathrm{SiC}$ различие между двумя квазикубическими позициями, $k 1$ и $k 2$, проявляется при рассмотрении третьей координационной сферы. Внизу на рис. 1 показано определение лабораторной системы координат, связанной с кристаллической симметрией, используемой в этой работе. Показаны возможные позиции донора азота; для $4 H$-SiC показана структура спинового центра $V 2$ со спином $S=3 / 2$, который является одним из основных объектов для применений, более того, именно на этом центре реализована возможность регистрации магнитного резонанса на одиночном центре при комнатной температуре [30]. Следует подчеркнуть, что впервые подобные центры наблюдались методом ЭПР Вайнером и Ильиным и были обозначены в кристалле $6 \mathrm{H}-\mathrm{SiC}$ как P3-, Р5-центры [31].
Одной из основных проблем использования $\mathrm{N} V$-центров в алмазе в системах квантовых вычислений и сенсорике является регулирование концентрации парамагнитных доноров азота, спиновые взаимодействия с которыми вызывают потерю когерентных свойств $\mathrm{N} V$-центров. Оказалось, что эта проблема имеется и в $\mathrm{SiC}$, так как азот $(\mathrm{N})$ является основной неконтролируемой примесью в этом материале. Более того, эта проблема даже серьезнее в $\mathrm{SiC}$ по сравнению с алмазом, так как в алмазе доноры азота характеризуются глубокими уровнями в запрещенной зоне, то есть достаточно сильно локализованы, тогда как доноры азота в $\mathrm{SiC}$ являются мелкими, в результате их волновые функции сильно делокализованы. В алмазе неспаренный донорный электрон делокализован только на атомах углерода, в случае карбида кремния возможна делокализация по углеродам и кремниям и, как оказалось, соотношение степени этой делокализации зависит от политипа и положения донорной примеси в кристаллической решетке $\mathrm{SiC}$.

Для $\mathrm{N} V$-центров в алмазе наблюдались эффекты кросс-релаксации (КР) между спиновой системой $\mathrm{N} V$-центров, с одной стороны, и другими дефектами в парамагнитном состоянии, например, соседними $\mathrm{N} V$-центрами с отличной ориентацией или с донорами азота, с другой стороны. Эти эффекты наблюдались как в ОДМР, так и при регистрации антипересечения спиновых подуровней. В настоящей работе нам удалось зарегистрировать сигналы кросс-релаксации между так называемыми $V 2$-спиновыми центрами в $4 H-\mathrm{SiC}$ и другим типом парамагнитных дефектов. Структура $V$ 2-центра, который широко обсуждался в литературе (см. например $[10,13,15])$, показана на рис. 1 для политипа $4 H-\mathrm{SiC}$ (спиновые центры с подобной структурой наблюдались и в политипе $6 H-\mathrm{SiC})$. Для наблюдения явления кроссрелаксации необходимо наличие по крайней мере двух спиновых систем с разными спиновыми температурами. В данном случае имеются системы с оптически выстроенными (поляризованными) населенностями спиновых подуровней спинового центра $V 2$ со спином $S=3 / 2$, с одной стороны, и неким парамагнитным дефектом, с другой стороны. Таким дефектом может быть центр $V 1$, который по структуре принадлежит к тому же семейству, что и $V 2$, но в отличие от $V 2$ его спиновые уровни практически не выстраиваются оптически и поэтому их спиновая температура отличается от спиновой температуры оптически выстроенных $V 2$-центров. Также возможна кросс-релаксация с донорами азота, так как для доноров азота со спином $S=1 / 2$ существует больцмановское распределение населенностей спиновых подуровней, которое, естественно, имеет спиновую температуру, отличную от оптически созданной инверсной населенности для $V 2$-центра.

На рис. 2 вверху показаны сигналы антипересечения спиновых подуровней, зарегистрированные по изменению интенсивности ИК-люминесценции $(850-900 \mathrm{~nm})$ на $V 2$-центрах в $4 H$-SiC для двух близких ориентаций магнитного поля относительно оси с симметрии 


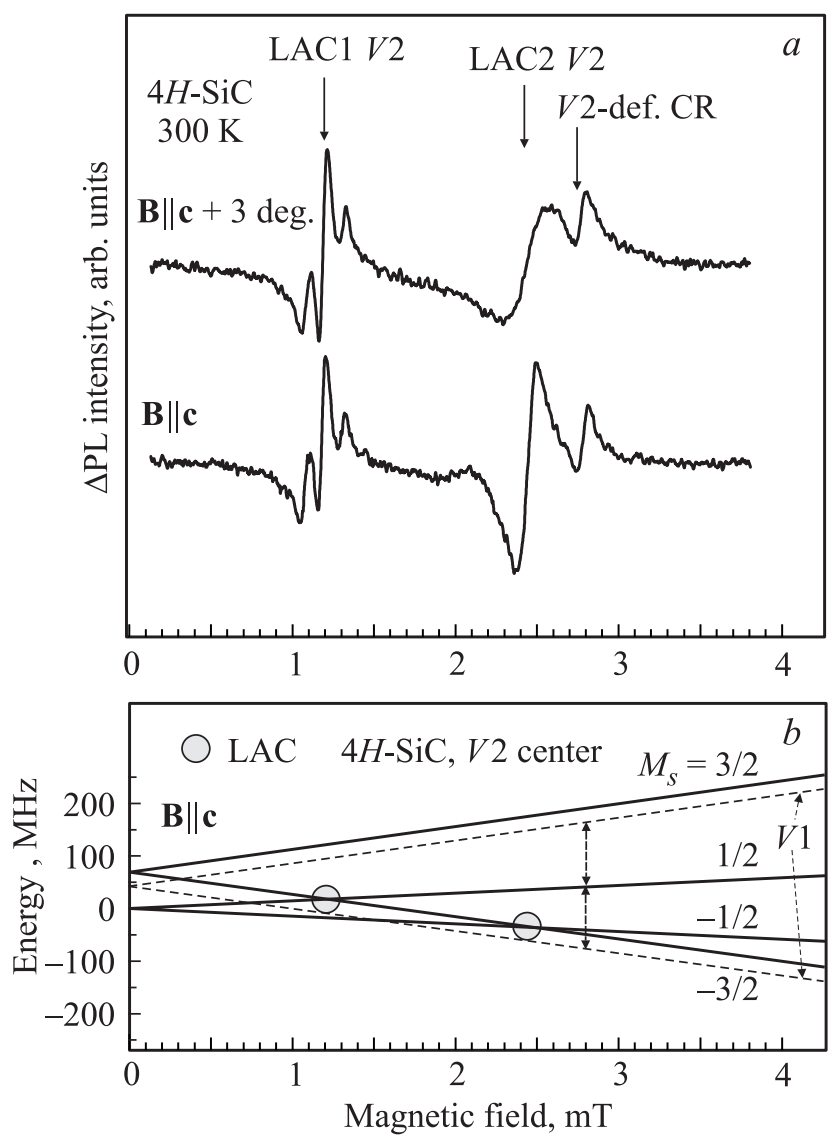

Рис. 2. а) Изменение интенсивности люминесценции $V 2$-центров в кристалле $4 H-\mathrm{SiC}$ в области антипересечения спиновых подуровней (LAC) при комнатной температуре, приведенные для двух ориентаций магнитного поля относительно оси с кристалла. Люминесценция регистрируется в области $850-900 \mathrm{~nm}$, длина волны возбуждения $805 \mathrm{~nm}$. b) Схема энергетических уровней в области антипересечения спиновых подуровней. Пунктиром показаны спиновые уровни $V 1$-центра с $M_{S}= \pm 3 / 2$ в магнитном поле (уровни с $M_{S}= \pm 1 / 2$ совпадают с уровнями $V 2$-центра). Пунктирными стрелками показано совпадение расстояний между уровнями в магнитном поле, в котором видна дополнительная линия.

кристалла: параллельной оси $(\mathbf{B} \| \mathbf{c})$ и отклоненной примерно на 3 градуса от оси с. Люминесценция возбуждалась лазером с длиной волны $805 \mathrm{~nm}$. Внизу приведены энергетические спиновые уровни для $V 2$-спинового центра, посчитанные для ориентации $\mathbf{B} \| \mathbf{c}$, поясняющие природу сигналов антипересечения уровней. Для регистрации сигналов АПУ использовалась модуляция магнитного поля на низкой частоте $(80 \mathrm{~Hz})$ с амплитудой 0.01-0.1 mT, а также подавалось постоянное магнитное поле, которое разворачивалось для создания условий АПУ. Сигнал в виде производной регистрировался с помощью синхронного детектора. Наряду с сигналами АПУ видна дополнительная линия, которую мы приписываем сигналу кросс-релаксации между спиновыми подуровнями $V 2$-центра и другим парамагнитным де- фектом. Известны следующие характеристики для возможных парамагнитных систем, взаимодействующих с $V 2$-центром, у которого $S=3 / 2$, расщепление тонкой структуры $\Delta=66 \mathrm{MHz}$, так как $\Delta=2 D(D-$ параметр тонкой структуры для центра с аксиальной симметрией), положение в магнитном поле первого и второго антикроссинга равно $D /\left(g \mu_{B}\right)$ и $2 D /\left(g \mu_{B}\right)$ соответственно, где используются стандартные обозначения. Для $V 1$-центра $\Delta=39 \mathrm{MHz}$; для мелкого донора азота в квазикубическом положении $\left(\mathrm{N}_{k}\right)$ константа сверхтонкого СТ-взаимодействия практически изотропна, $A=51 \mathrm{MHz}$, для гексагонального положения $\left(\mathrm{N}_{h}\right) A \cong 3 \mathrm{MHz}$; для всех центров электронные $g$-факторы близки к 2.00 . Таким образом, совпадение расщеплений спиновых подуровней каждого из центров в магнитном поле может происходить в области наблюдения линии, обозначенной на рис. 2 как $V 2$-def. CR (CR - cross-relaxation). В качестве возможного объяснения дополнительной линии на рис. 2 пунктиром показаны спиновые уровни $V 1$-центра с $M_{S}= \pm 3 / 2$ в магнитном поле (уровни с $M_{S}= \pm 1 / 2$ совпадают с уровнями $V 2$-центра). Пунктирными стрелками показано совпадение расстояний между уровнями в магнитном поле, в котором видна дополнительная линия. Эта линия лежит в пределах сигнала АПУ для $V 2$-центра, так что возможен обмен энергией между этими системами, приводящий к изменению населенностей этих уровней и, как следствие, к изменению интенсивности ИК-люминесценции.

В работах по $\mathrm{N} V$-центрам в алмазе взаимодействию с донорами азота отводится не только отрицательная роль (уменьшение времени когерентности), но и положительная, так как спиновая система азота может быть использована для возможной записи когерентной информации в квантовых вычислениях. Для мелких доноров азота в $\mathrm{SiC}$ такие условия могут быть созданы только при достаточно низких температурах, так как при комнатной температуре мелкие доноры ионизируются. Последнее обстоятельство может стимулировать релаксационные процессы из-за взаимодействия неспаренных электронов спинового центра $(V 2)$ со спинами электронов в зоне проводимости, что должно привести к укорачиванию времен релаксации. Величина этого взаимодействия в значительной степени определяется пространственным распределением плотности волновой функции неспаренного электрона донора азота.

Задачей настоящей работы является получение информации об электронной структуре мелких доноров азота на примере двух основных политипов $\mathrm{SiC}$ и пространственном распределении спиновой плотности неспаренного электрона. При этом будут проанализированы данные, ранее полученные авторами работы и имеющиеся результаты других исследований. Важно подчеркнуть, что имеется существенное разночтение в интерпретации СТ-взаимодействий с лигандными атомами, поскольку в более поздних работах были обнаружены сильные СТ-взаимодействия с ближайшими лигандными атомами 
кремния и углерода, которые непосредственно проявились в виде сателлитов в спектрах ЭПР, поэтому эти взаимодействия не наблюдались в спектрах двойного электронно-ядерного резонанса (ДЭЯР). В настоящей работе мы попытаемся связать результаты ЭПР и ДЭЯР для построения самосогласованной электронной структуры доноров азота.

\section{2. Доноры азота}

2.1. В ы с о к ч ч с т о тный ЭПР-ДЭЯР. Важным вопросом является пространственная делокализация электронной волновой функции донорных центров в полупроводниках. Основным методом для получения этой информации является спектроскопия двойного электронно-ядерного резонанса (ДЭЯР), разработанная в работе [32], которая была применена к донорным примесям в $\mathrm{Si}$ [32-34], а также к акцепторным примесям в $\mathrm{SiC}$ [35]. В этих экспериментах определено СТ-взаимодействие между неспаренным электронным спином донора и ядерными спинами окружающих атомов, которое затем преобразовывается в спиновую плотность электронной волновой функции на различных атомных позициях согласно выражению

$$
A_{i}=(8 \pi / 3) g_{e} \mu_{B} g_{n i} \mu_{N}\left|\Psi\left(\mathbf{r}_{i}\right)\right|^{2} .
$$

Здесь $A_{i}$ - константа изотропного СТ-взаимодействия с $i$-м лигандным атомом с ядерным $g$-фактором $g_{n i}$, $g_{e}-$ электронный $g$-фактор, $\mu_{B}$ и $\mu_{N}-$ магнетон Бора и ядерный магнетон соответственно; $\left|\Psi\left(\mathbf{r}_{i}\right)\right|^{2}-$ плотность волновой функции неспаренного электрона на $i$-м лигандном ядре.

В настоящей работе будут представлены результаты исследований ЭПР и ДЭЯР доноров $\mathrm{N}$ в $4 H-\mathrm{SiC}$ и 6H-SiC. Ранее доноры N были исследованы методами оптического поглощения, эмиссионной спектроскопии и комбинационного рассеяния [36,37]. Первые исследования ЭПР на донорах $\mathrm{N}$ в $\mathrm{SiC}$ были сделаны в работе [38] и первые измерения ДЭЯР - при $9.5 \mathrm{GHz}$ в [39,40]. Исследования высокочастотного ЭПР при $142 \mathrm{GHz}$ в [41] позволили разделить перекрывающиеся линии ЭПР благодаря высокому спектральному разрешению и приписать различные линии ЭПР конкретным центрам (узлам) в решетке $\mathrm{SiC}$. Дополнительные измерения ДЭЯР представлены в [42], и обзор электронных свойств доноров $\mathrm{N}$, полученный методами ЭПР и ДЭЯР при $9.5 \mathrm{GHz}$, представлен в [43].

Интерпретация данных ДЭЯР для донора N в $\mathrm{SiC}$ составляет значительную проблему, так как приписывание линий ДЭЯР определенной координационной сфере и конкретному атому затруднительно, поскольку волновая функция донорных электронов представляет собой линейную комбинацию волновых функций в шести минимумах зоны проводимости. Вследствие этого происходит эффект интерференции, который приводит к тому, что плотность волновой функции не затухает монотонно с расстоянием от положения донора. Путем сравнения спектров ДЭЯР с теоретическими расчетами распределения спиновой плотности, основанными на теории работ $[44,45]$, в работе [46] были приписаны линии ДЭЯР определенным лигандным атомам в кристаллической решетке $\mathrm{SiC}$, а также были объяснены различия в поведении донора $\mathrm{N}$ в $k$-позиции в $4 H-\mathrm{SiC}$ и $h$-, $k 1$ - и $k 2$-позициях в $6 H$-SiC. При этом использовалось также сходство сигналов ДЭЯР для $k$-позиции доноров $\mathrm{N}$ в $4 H-\mathrm{SiC}$ и доноров $\mathrm{P}(\mathrm{As}, \mathrm{Sb})$ в кремнии [32].

Важным аспектом исследования ДЭЯР [46] является то, что они выполнены на высокой микроволновой частоте $95 \mathrm{GHZ}$ ( $W$-диапазон), что приводит к высокому спектральному разрешению как в спектрах ЭПР, так и в спектрах ДЭЯР: в спектрах ЭПР разделяются сигналы доноров азота, находящиеся в разных позициях кристаллической решетки; в спектрах ДЭЯР разделяются сигналы ядер ${ }^{13} \mathrm{C}$ (природное содержание $1.11 \%, I=1 / 2$ ) и ${ }^{29} \mathrm{Si}$ (природное содержание $4.67 \%, I=1 / 2$ ).

Теоретические и практические исследования СТвзаимодействия в легированных азотом кристаллах $4 H$ и $6 H$-SiC были представлены недавно в работе [47]. Были рассчитаны СТ-тензоры в рамках теории функционала плотности и проанализированы результаты работ [46,48-50]. В работе [51] было изучено зондирование волновых функций мелких доноров $\mathrm{N}$ в кристаллах $\mathrm{SiC} \mathrm{c}$ измененным изотопным составом методом ЭПР. Недавний прогресс в исследовании электронной структуры мелких доноров азота и фосфора в $3 C$-, $4 H$ - и $6 H-\mathrm{SiC}$ был рассмотрен в [52].

На рис. 3 показаны зарегистрированные с помощью высокочастотного электронного спинового эха (ЭСЭ) спектры ЭПР доноров $\mathrm{N}$ в необогащенном и обогащенном изотопом ${ }^{13} \mathrm{C} 6 H-\mathrm{SiC}$ (верхняя панель) и $4 H-\mathrm{SiC}$ (нижняя панель), измеренные при $1.2 \mathrm{~K}$ и $95 \mathrm{GHz}$ для двух различных ориентаций магнитного поля в кристалле: магнитное поле параллельно с-оси $(\mathbf{B} \| \mathbf{c})$ и магнитное поле в плоскости, перпендикулярной к оси c $(\mathbf{B} \perp \mathbf{c})$. Центральные СТ-линии различных сигналов ЭПР маркируются, используя следующие аббревиатуры: $\mathrm{N} k$ для донора $\mathrm{N}$ в квазикубической позиции в кристалле $4 H-\mathrm{SiC}$ и $\mathrm{N} k 1$ и $\mathrm{N} k 2$ для донора $\mathrm{N}$ в квазикубических позициях $k 1$ или $k 2$ в кристалле $6 H-\mathrm{SiC}$ соответственно. $\mathrm{N} h$ указывает на сигнал, связанный с $\mathrm{N}$ в гексагональной позиции в кристаллах $4 H-\mathrm{SiC}$ или 6H-SiC (рис. 1). Из-за СТ-взаимодействия неспаренного электрона с ядром донора $\mathrm{N}$ линии ЭПР расщепляются на три компоненты (изотоп ${ }^{14} \mathrm{~N}$, природное содержание $99.63 \%, I=1)$. Для $h$-позиции расщепление слишком мало, чтобы быть разрешенным в спектрах ЭПР. Для $\mathrm{N} k$ в $4 H$-SiC расщепление составляет $1.9 \mathrm{mT}$, а для $\mathrm{N} k 1$ и $\mathrm{N} k 2$ в $6 H-\mathrm{SiC}$ расщепление составляет 1.23 и $1.25 \mathrm{mT}$ соответственно. Экспериментальные значения $g$-фактора и СТ-взаимодействия для донора $\mathrm{N}$ согласуются со значениями, найденными в литературе (табл. 1).

Из рис. 3 видно, что спектры ЭПР в результате обогащения изотопом ${ }^{13} \mathrm{C}$ сильно отличаются для 


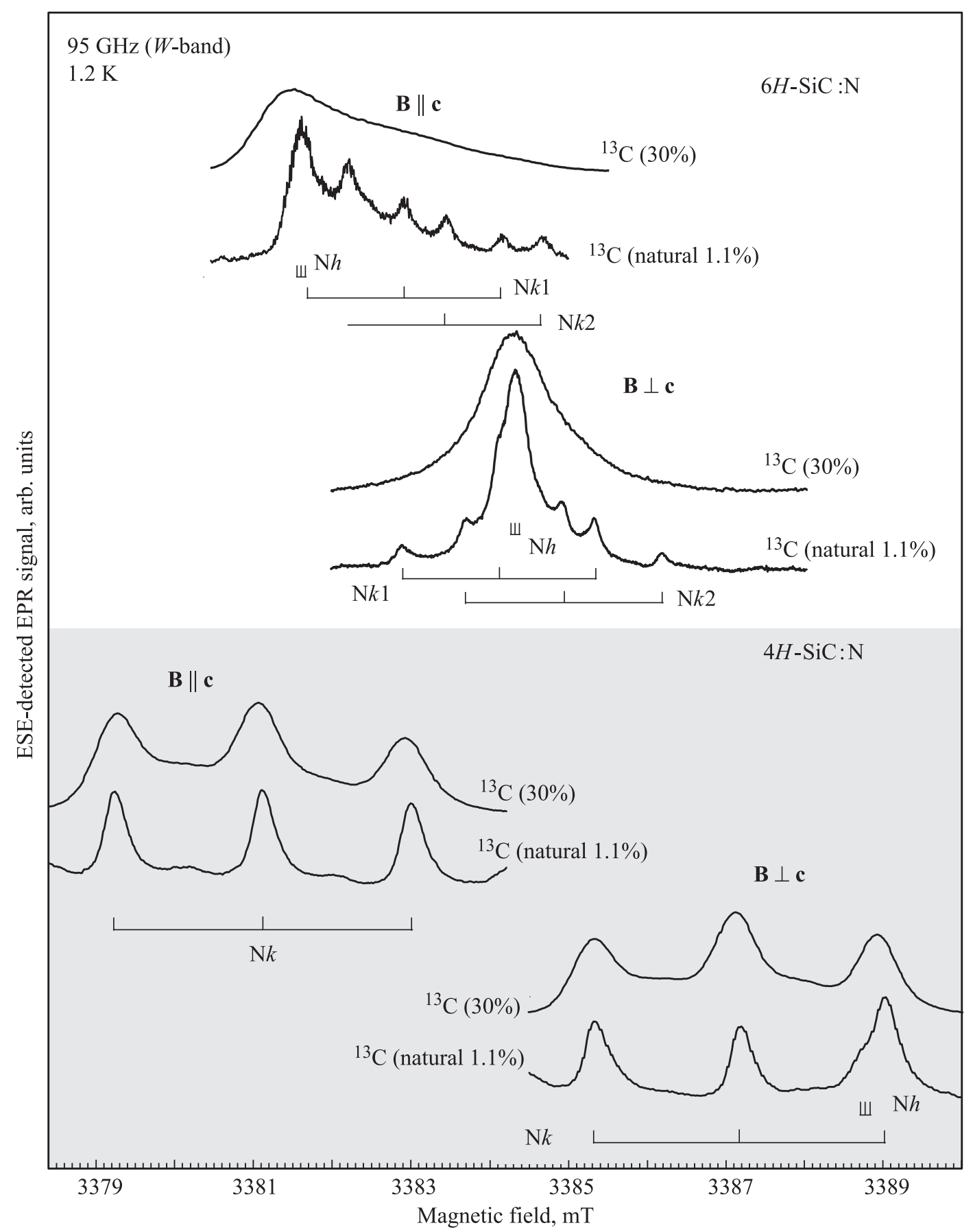

Рис. 3. Спектры ЭПР, детектируемые методом ЭСЭ, донора $\mathrm{N}$ в кристаллах $4 H-\mathrm{SiC}$ и $6 H$-SiC: (необогащенном и обогащенном изотопом $\left.{ }^{13} \mathrm{C}\right)$, зарегистрированные при температуре $1.2 \mathrm{~K}$ и частоте $95 \mathrm{GHz}$ для двух различных ориентаций магнитного поля в кристалле. На верхних рисунках магнитное поле параллельно с-оси $(\mathbf{B} \| \mathbf{c})$, на нижних рисунках магнитное поле перпендикулярно $\mathbf{c}-о$ оси $(\mathbf{B} \perp \mathbf{c})$. В каждой панели верхний спектр связан с образцом, обогащенным изотопом ${ }^{13} \mathrm{C}$, а нижний $-\mathrm{c}$ необогащенным образцом. Ширина линии указывается для каждого спектра. Из трех сверхтонких линий ЭПР, связанных с квазикубическими позициями центральная линия обозначается $\mathrm{N} k(4 H-\mathrm{SiC}), \mathrm{N} k 1$ или $\mathrm{N} k 2(6 H-\mathrm{SiC})$. Сверхтонкое расщепление линии ЭПР в позиции $h$ слишком мало, чтобы быть разрешенным в спектре ЭПР. Одна линия, связанная с позицией $h$, обозначается $\mathrm{N} h$, но едва видна в спектре.

двух политипов. Ширины линий ЭПР в спектрах, зарегистрированных по ЭСЭ, не всегда точно отражают СТ-взаимодействия с лигандами, эти ширины будут более детально исследованы методом непрерывного
(CW - continuous wave) ЭПР в следующем параграфе, но тем не менее влияние изменения содержания изотопов проявляется и в спектрах ЭСЭ. В $4 H-\mathrm{SiC}$ наблюдается небольшое уширение линии ЭПР, в то 
Таблица 1. Экспериментальные характеристики мелких доноров $\mathrm{N}$ в кристаллах $3 C$-SiC, $4 H$-SiC и $6 H$ - $\mathrm{SiC}$ в различных позициях из $[42,45,50]:$ энергии ионизации, долинно-орбитальное расщепление, $g$-факторы, изотропные $(a)$ и анизотропные $(b)$ константы сверхтонкого взаимодействия и соответствующие им спиновые плотности для $s$ - и $p$-электронов на $\mathrm{N}$, квадрупольные параметры $q$. Для сравнения представлены аналогичные параметры для мелких доноров Р и As в кремнии [31]

\begin{tabular}{|c|c|c|c|c|c|c|c|c|c|}
\hline Кристалл & Позиция & $\begin{array}{c}\text { Энергия ионизации } \\
E_{g} / \text { долинно-орбитальное } \\
\text { расщепление }(\mathrm{meV})\end{array}$ & $g_{\|}$ & $g_{\perp}$ & $\begin{array}{c}a \\
(\mathrm{MHz})\end{array}$ & $\begin{array}{c}b \\
(\mathrm{MHz})\end{array}$ & $\begin{array}{c}q \\
(\mathrm{MHz})\end{array}$ & $\begin{array}{c}s \\
(\%)\end{array}$ & $\begin{array}{c}p \\
(\%)\end{array}$ \\
\hline $3 C-\mathrm{SiC}$ & $k$ & $54 / 8.37$ & 2.0050 & 2.0050 & 3.5 & & & 0.19 & \\
\hline $4 H-\mathrm{SiC}$ & $\begin{array}{l}h \\
k\end{array}$ & $\begin{array}{c}52.1 / 7.6 \\
91.8 / 45.5\end{array}$ & $\begin{array}{l}2.0055 \\
2.0010\end{array}$ & $\begin{array}{l}2.0043 \\
2.0013\end{array}$ & $\begin{array}{c}2.9 \\
50.97\end{array}$ & $\begin{array}{l}0.080 \\
0.004\end{array}$ & $\begin{array}{l}\cdots \\
\sim 0\end{array}$ & $\begin{array}{l}0.16 \\
2.8\end{array}$ & $\begin{array}{l}0.14 \\
0.007\end{array}$ \\
\hline $6 H-\mathrm{SiC}$ & $\begin{array}{c}h \\
k 1 \\
k 2\end{array}$ & $\begin{array}{c}81 / 12.6 \\
137.6 / 60.3 \\
142.4 / 62.6\end{array}$ & $\begin{array}{l}2.0048 \\
2.0040 \\
2.0037\end{array}$ & $\begin{array}{l}2.0028 \\
2.0026 \\
2.0030\end{array}$ & $\begin{array}{c}2.52 \\
33.221 \\
33.564\end{array}$ & $\begin{array}{l}0.12 \\
0.004 \\
0.009\end{array}$ & $\begin{array}{l}0.019 \\
0.007 \\
0.007\end{array}$ & $\begin{array}{l}0.14 \\
1.83 \\
1.85\end{array}$ & $\begin{array}{l}0.22 \\
0.007 \\
0.016\end{array}$ \\
\hline \multicolumn{10}{|l|}{$\mathrm{Si}$} \\
\hline $\mathrm{P}$ & & $44 / 11.7$ & 1.9985 & & 117.53 & & & 0.9 & \\
\hline As & & $49 / 21.2$ & 1.9984 & & 198.35 & & & 1.35 & \\
\hline
\end{tabular}

же время в $6 H$-SiC ширина линии увеличивается более чем в 3 раза. Из этих результатов можно сделать предварительный вывод, что в $4 H$-SiC основная часть спиновой плотности локализуется на атомах $\mathrm{Si}$, так как $30 \%$ обогащение ${ }^{13} \mathrm{C}$ приводит к небольшому уширению линий. В противоположность этому, в $6 H-\mathrm{SiC}$ мы приходим к выводу, что основная часть спиновой плотности донора $\mathrm{N}$ локализуется на атомах C из-за большого уширения линии при $30 \%$ обогащении ${ }^{13} \mathrm{C}$. Далее мы покажем, что найдено разумное значение для наблюдаемой ширины линии по имеющимся данным ДЭЯР ${ }^{13} \mathrm{C}$ и ${ }^{29} \mathrm{Si}$.

На рис. 4, $а$ показаны спектры ДЭЯР, зарегистрированные по ЭСЭ для ядер ${ }^{29} \mathrm{Si}$ и ${ }^{13} \mathrm{C}$ в $4 H-\mathrm{Si}^{13} \mathrm{C}$ и $6 \mathrm{H}-\mathrm{Si}^{13} \mathrm{C}$ для В $\| \mathbf{c}$ при $1.2 \mathrm{~K}$ и $95 \mathrm{GHz}$. Показан только спектр на высокочастотной стороне зеемановской частоты. Верхний спектр принадлежит донору $\mathrm{N} k$ в $4 H-\mathrm{SiC}$. Положения линий отмечены ромбиками. Средний спектр был измерен для $\mathrm{N} k 1$ по линии ЭПР $(B=3382.2 \mathrm{mT}$, см. рис. 3), нижний спектр для $\mathrm{N} k 2(B=3381.5 \mathrm{mT})$ в $6 H$-SiC. Линии $\mathrm{N} k 1$ и $\mathrm{N} k 2$ для ${ }^{29} \mathrm{Si}$ отмечены квадратиками, положение линий $\mathrm{N} h$ обозначены кружками. Положения линий $\mathrm{N} h$ для ${ }^{13} \mathrm{C}$ отмечены открытыми кружками, те же линии для $\mathrm{N} k 1$ - квадратами и для $\mathrm{N} k 2$ - треугольниками. Стрелками обозначена зеемановская частота ядер ${ }^{29} \mathrm{Si}$ и ${ }^{13} \mathrm{C}$.

Из сравнения спектров ДЭЯР ${ }^{29} \mathrm{Si}$ и ${ }^{13} \mathrm{C}$ для различных позиций донора азота в $4 H-\mathrm{Si}^{13} \mathrm{C}$ и $6 H-\mathrm{Si}^{13} \mathrm{C}$ (рис. $4, a)$ ясно, что распределение спиновой плотности электрона донора $\mathrm{N}$ по ядрам ${ }^{13} \mathrm{C}$ и ${ }^{29} \mathrm{Si}$ отличается для $4 H-\mathrm{SiC}$ и $6 H$-SiC. В то время как основная часть спиновой плотности, как и ожидалось для донорного электрона в $4 H-\mathrm{SiC}$, локализуется на атомах кремния, в кристалле $6 H$-SiC спиновая плотность неспаренного электрона донора азота локализована главным образом на атомах углерода. Также отметим, что спектр ДЭЯР в кристалле $6 H$-SiC содержит больше линий вблизи зеемановской частоты ядра ${ }^{13} \mathrm{C}$, чем спектр $4 H-\mathrm{SiC}$, как вблизи зеемановской частоты ${ }^{13} \mathrm{C}$, так и вблизи зеемановской частоты ${ }^{29} \mathrm{Si}$.

Спектры ЭПР и ДЭЯР для доноров $\mathrm{N}$ в $\mathrm{SiC}$ могут быть описаны спиновым гамильтонианом следующего вида [46]:

$$
\begin{aligned}
H= & \mu_{B} \mathbf{S} \cdot \stackrel{\leftrightarrow}{g}_{\mathrm{N}} \cdot \mathbf{B}+\left(\mathbf{S} \cdot \overleftrightarrow{A}_{\mathrm{N}}-\gamma_{\mathrm{N}} \mathbf{B}\right) \cdot \mathbf{I}_{\mathrm{N}} \\
& +\mathbf{I}_{\mathrm{N}} \cdot \overleftrightarrow{P}_{\mathrm{N}} \cdot \mathbf{I}_{\mathrm{N}}+\sum\left(\mathbf{S} \cdot \overleftrightarrow{A}_{K}-\gamma_{\mathrm{K}} \mathbf{B}\right) \cdot \mathbf{I}_{\mathrm{K}},
\end{aligned}
$$

где $S=1 / 2, \overleftrightarrow{g}_{\mathrm{N}}-g$-тензор для донора азота, $\overleftrightarrow{A}_{\mathrm{N}}-$ тензор сверхтонкого взаимодействия с азотом ${ }^{14} \mathrm{~N}$ $(I=1)$ и $\overleftrightarrow{A}_{K}$ - тензор сверхтонкого взаимодействия с ядерными спинами ${ }^{13} \mathrm{C}(I=1 / 2)$ и ${ }^{29} \mathrm{Si}(I=1 / 2), \overleftrightarrow{P}_{\mathrm{N}}-$ тензор квадрупольного взаимодействия с азотом ${ }^{14} \mathrm{~N}$; $\gamma_{\mathrm{N}}$ - гиромагнитное отношение для ядра ${ }^{14} \mathrm{~N}, \gamma_{\mathrm{K}}-$ гиромагнитное отношение для ядер ${ }^{13} \mathrm{C}$ и ${ }^{29} \mathrm{Si}$. Учитывая, что тензоры сверхтонкого взаимодействия имеют симметрию близкую к аксиальной, можно переписать их как $A_{z z}=a+2 b, A_{y y}=a-b-b^{\prime}$ и $A_{x x}=a-b+b^{\prime}$ (параметр $b^{\prime}$ характеризует отклонение от аксиальной симметрии) [53]. В системе главных осей квадрупольное взаимодействие имеет вид для аксиальной симметрии, $P_{\mathrm{N}}\left(I_{z}^{2}-1 / 3 I^{2}\right)$ с $P_{\mathrm{N}}=3 / 2 P_{z z}=3 q$ и $P_{x x}=P_{y y}=-q$. Отклонение от аксиальной симметрии описывается как $q^{\prime}=1 / 2\left(P_{x x}-P_{y y}\right), P_{x x}=-q+q^{\prime}$ и $P_{y y}=-q-q^{\prime}$.

Угол $\theta$ определяется как угол между магнитным полем и с-осью, $\phi$-угол в плоскости, перпендикулярной с-оси. Лабораторная система осей ориентирована следующим образом. Плоскость (112̄0) эквивалентна плоскости $x z$. Ось $z$ (ось [0001]) параллельна кристаллографической с-оси, и $\theta=0^{\circ}$ и $\phi=0^{\circ}$. Ось $z$ (ось $\left.[\overline{1} 100]\right)$ лежит в плоскости $(1 \overline{1} \overline{2} 0)$, перпендикулярной к оси с 

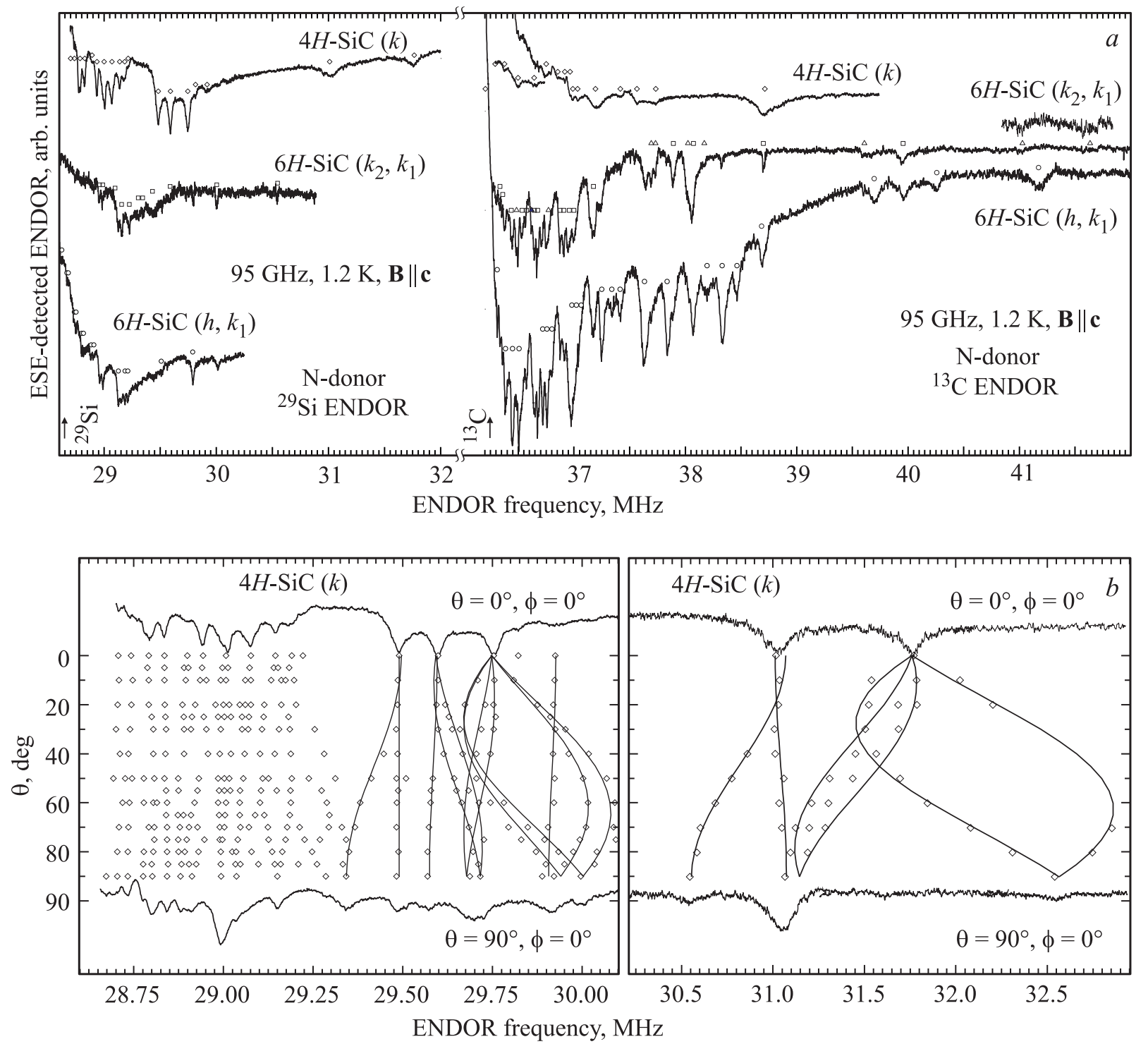

Рис. 4. а) Спектры ДЭЯР на ядрах ${ }^{29} \mathrm{Si}$ и ${ }^{13} \mathrm{C}$ для доноров азота в разных позициях в $4 H-\mathrm{Si}^{13} \mathrm{C}$ и $6 H$-Si ${ }^{13} \mathrm{C}$, зарегистрированные в ориентации В $\|$ с при температуре $1.2 \mathrm{~K}$, частоте $95 \mathrm{GHz}$. Показан только спектр на высокочастотной стороне от зеемановской частоты. Верхний спектр принадлежит донору $\mathrm{N}$, занимающему позицию $k$ в $4 H$-SiC. Положения линий были отмечены ромбиками. Средний спектр был зарегистрирован для $\mathrm{N}$ в позиции $k 1$ по спектру ЭПР $(B=3382.2 \mathrm{mT}$, см. рис. 3$)$, нижний спектр для $\mathrm{N}$ в позиции $k 2(B=3381.5 \mathrm{mT})$ в $6 H$-SiC. Линии для $\mathrm{N}$ в $k 1-, k 2$-позициях (взаимодействие с ядрами $\left.{ }^{29} \mathrm{Si}\right)$ отмечены квадратиками, положения линий $h$-позиций обозначены кружками. Положения линий для $\mathrm{N}$ в $h$-позиции (ядра $\left.{ }^{13} \mathrm{C}\right)$ отмечены открытыми кружками, положения линий для $\mathrm{N}$ в $k 1$-позиции $\left(\right.$ ядра $\left.{ }^{13} \mathrm{C}\right)-$ квадратами и положения линий для $\mathrm{N}$ в $k 2$-позиции (ядра $\left.{ }^{13} \mathrm{C}\right)-$ треугольниками. Зеемановская частота ядер ${ }^{29} \mathrm{Si}$ и ${ }^{13} \mathrm{C}$ обозначена стрелками. $b$ ) Ориентационная зависимость линий ДЭЯР для $\mathrm{N}$ в k-позиции для ядер ${ }^{29} \mathrm{Si}$ (точки экспериментальных данных отмечены ромбиками) в 4H-SiC в плоскости $(11-20)$ или $(z x)$. Спектры были откорректированы с поправкой на смещение ядерной зеемановской частоты относительно ориентации В $\| \mathbf{c}(\theta=0)$.

$\left(\theta=90^{\circ}\right.$ и $\left.\phi=0^{\circ}\right)$. Ось $y$ (ось $\left.[11 \overline{2} 0]\right)$ перпендикулярна оси х в плоскости, перпендикулярной к оси с $\left(\theta=90^{\circ}\right.$ и $\left.\phi=90^{\circ}\right)($ см. также рис. 1).

На рис. $4, b$ представлена ориентационная зависимость ДЭЯР ${ }^{29} \mathrm{Si}$ для $\mathrm{N} k$ в $4 H-\mathrm{SiC}$ в плоскости $(11 \overline{2} 0)(z x)$, спектры ДЭЯР были откорректированы по сдвигу ядерной зеемановской частоты по отношению к ориентации В $\|$ с из-за сдвига линии ЭПР в магнитном поле, которые связаны с анизотропией $g$-фактора. На рис. $4, b$ приведены экспериментальные результаты для всех измеренных ориентаций для $\mathrm{N} k$, а также рассчитанные ориентационные зависимости спектров ДЭЯР в соответствие со спиновым гамильтонианом (2). В целом, были определены шестнадцать различных групп ядер, которые представлены в [46], для которых были рассчитаны значения СТ-взаимодействия и углы Эйлера.

После добавления результатов новых исследований [48-52], где в спектрах ЭПР непосредственно на- 
Таблица 2. Основанные на расчетных данных ДЭЯР, $\Delta B(\mathrm{Si}), \Delta B(\mathrm{C})$ и ширины линий ЭПР, зарегистрированные методом ЭСЭ в $W$-диапазоне, $\Delta B$ (экспериментальная) (в $\mathrm{mT}$ ) для различных позиций, а также общая расчетная ширина линии $\Delta B$ (суммарная) для природных образцов, а также обогащенных изотопом ${ }^{13} \mathrm{C} 4 H$-SiC и $6 H$-SiC. Все значения указаны в mT. Различное содержание изотопов указано в тексте. В обогащенном образце $6 H-\mathrm{SiC}$ не представлялось возможным провести различие между экспериментальными ширинами линии $\Delta B$ (экспериментальная) в трех позициях. Расчетное значение $1 \mathrm{mT}$ дается для всех трех позиций. Предполагаемая погрешность в других экспериментальных ширинах линии составляет $0.05 \mathrm{mT}$

\begin{tabular}{|c|c|c|c|c|c|c|c|}
\hline \multirow{2}{*}{ Политип } & \multirow{2}{*}{$\begin{array}{c}\Delta B(\mathrm{Si}) \\
\text { природный }\end{array}$} & \multicolumn{2}{|c|}{$\Delta B(\mathrm{C})$} & \multicolumn{2}{|c|}{$\begin{array}{c}\Delta B \\
\text { (суммарная) }\end{array}$} & \multicolumn{2}{|c|}{$\begin{array}{c}\Delta B \\
\text { (экспериментальная) }\end{array}$} \\
\hline & & природный & обогащенный & природный & обогащенный & природный & обогащенный \\
\hline $4 H-\mathrm{SiC}, \mathrm{N} k$ & 0.194 & 0.084 & 0.43 & 0.28 & 0.62 & 0.32 & 0.57 \\
\hline $6 H-\mathrm{SiC}, \mathrm{N} h$ & 0.063 & 0.176 & 0.99 & 0.26 & 1.05 & 0.30 & $\sim 1$ \\
\hline $6 H-\mathrm{SiC}, \mathrm{N} k 1$ & 0.066 & 0.102 & 0.58 & 0.17 & 0.64 & 0.25 & $\sim 1$ \\
\hline $6 H-\mathrm{SiC}, \mathrm{N} k 2$ & 0.092 & 0.165 & 0.88 & 0.25 & 0.97 & 0.30 & $\sim 1$ \\
\hline
\end{tabular}

блюдались сильные СТ-взаимодействия с некоторыми атомами в первой и второй координационных сферах $\mathrm{Si}$ и $\mathrm{C}$, результаты ДЭЯР были пересмотрены в настоящей работе, и эти взаимодействия следует исключить из финальных таблиц, представленных в работе [46].

Сначала мы обсудим результаты исследований ЭПР и ДЭЯР изотропного и анизотропного СТ-взаимодействий с ядром ${ }^{14} \mathrm{~N}$ для доноров $\mathrm{N}$ в основных политипах $\mathrm{SiC}$ : $3 C, 4 H$ и $6 H$, которые представлены в табл. 1 . Значения взяты из $[43,46]$. Только для $h$-позиции в $6 H-\mathrm{SiC}$ нами обнаружены небольшие различия в параметрах (табл. 1). Остальные значения совпадают в пределах погрешности эксперимента и не представлены в таблице. В табл. 1 приведены экспериментальные значения энергии ионизации доноров $\mathrm{N}$, долинно-орбитальное расщепление, $g$-факторы, изотропные $(a)$ и анизотропные $(b)$ константы СТ-взаимодействия и соответствующие им спиновые плотности для $s$ - и $p$-электронов на ${ }^{14} \mathrm{~N}$ в $3 C$, $4 \mathrm{H}$ и $6 H-\mathrm{SiC}$, а также квадрупольные взаимодействия $(q)$. Для сравнения те же самые параметры представлены для мелких доноров $\mathrm{P}$ и As в $\mathrm{Si}$. Там $g$-фактор и СТ-взаимодействия являются изотропными. Как видно из табл. 1, в общем случае значение энергии ионизации для различных позиций не коррелирует с изотропными СТ-взаимодействиями, т.е. со спиновой плотностью на ядрах N. Например, энергии ионизации для $k$-позиции в $4 H-\mathrm{SiC}$ и $h$-позиции в $6 H-\mathrm{SiC}$, примерно равны, но изотропное СТ-взаимодействие доноров азота для $k$-позиции в $4 H$-SiC примерно в 20 раз больше, чем для $h$-позиции в $6 H$-SiC.

2.2. ЭПР доноров азота в кристаллах с измененным содержанием изотопов кремния и углерода с ядерными магнитными моментами. На рис. 5, $a$ показаны спектры ЭПР мелких доноров азота в трех кристаллах $6 H-\mathrm{SiC}$ : с природным изотопным составом, обогащенным ${ }^{28} \mathrm{Si}\left(<0.5 \%{ }^{29} \mathrm{Si}\right)$ и обогащенным ${ }^{13} \mathrm{C}(\sim 25 \%)$. Спектры были измерены при $40 \mathrm{~K}$ в ориентации магнитного поля $\mathbf{B} \perp \mathbf{c}$.

Для азота в позициях $k 1, k 2$ наблюдается по три линии ЭПР, поскольку имеется только один изотоп азота ${ }^{14} \mathrm{~N}$ (распространенность 99.63\%) с ядерным спином $I=1$ (число линий равно $2 I+1$ ). Сигналы доноров $\mathrm{N}$ в позициях $k 1, k 2$ отличаются по параметрам, поэтому наблюдается расщепление линий. Высокополевые компоненты спектров ЭПР мелких доноров азота в $k 1$ и $k 2$-позициях в $6 H$-SiC, представленные на рис. 5, $a$, приведены отдельно на рис. 5, $b$ в увеличенном масштабе для ориентации $\mathbf{B} \perp \mathbf{c}$. Видно небольшое сужение линий ЭПР в результате уменьшения концентрации изотопа ${ }^{29} \mathrm{Si}$ на порядок, приводящее к лучшему разрешению сигналов для позиций азота $k 1$ и $k 2$. В то же время обогащение изотопом ${ }^{13} \mathrm{C}$ приводит к существенному уширению линий ЭПР. Ширины линий ЭПР, зарегистрированные в кристаллах с природным и измененным изотопным составом, представлены в табл. 2. На рис. 5, $b$ для кристалла с обедненным содержанием изотопа ${ }^{29} \mathrm{Si}$, видны дополнительные линии в виде сателлитов, которые расположены симметрично относительно центральной линии и указаны стрелками. Видно, что такие сателлиты присутствуют для каждого компонента СТ-структуры азота в позициях $k 1$ и $k 2$, причем расщепление между линиями практически не зависит от ориентации кристалла и равно $0.5 \mathrm{mT}$ $(14 \mathrm{MHz})$. Поскольку эти сателлиты зарегистрированы в кристаллах с обедненным содержанием изотопа ${ }^{29} \mathrm{Si}$ они могут быть обусловлены только СТ-взаимодействием с углеродом. В соответствии с природным содержанием ${ }^{13} \mathrm{C}$, соотношение интенсивностей свидетельствует о взаимодействии с тремя практически эквивалентными атомами углерода, причем это взаимодействие является изотропным в пределах экспериментальной ошибки и является одинаковым для позиций азота $k 1$ и $k 2$.

На рис. 5, с приведены спектры ЭПР мелких доноров азота в $k$-позиции в трех кристаллах $4 H-\mathrm{SiC}$ для ориентации В $\|$ с: с природным изотопным составом; обогащенных ${ }^{28} \mathrm{Si}$ и, таким образом, имеющих низкое содержание изотопа ${ }^{29} \mathrm{Si}$ (менее $0.5 \%$ ); и обогащенных изотопом ${ }^{13} \mathrm{C}(\sim 15 \%)$. Показаны только высокополевые компоненты спектров ЭПР. 


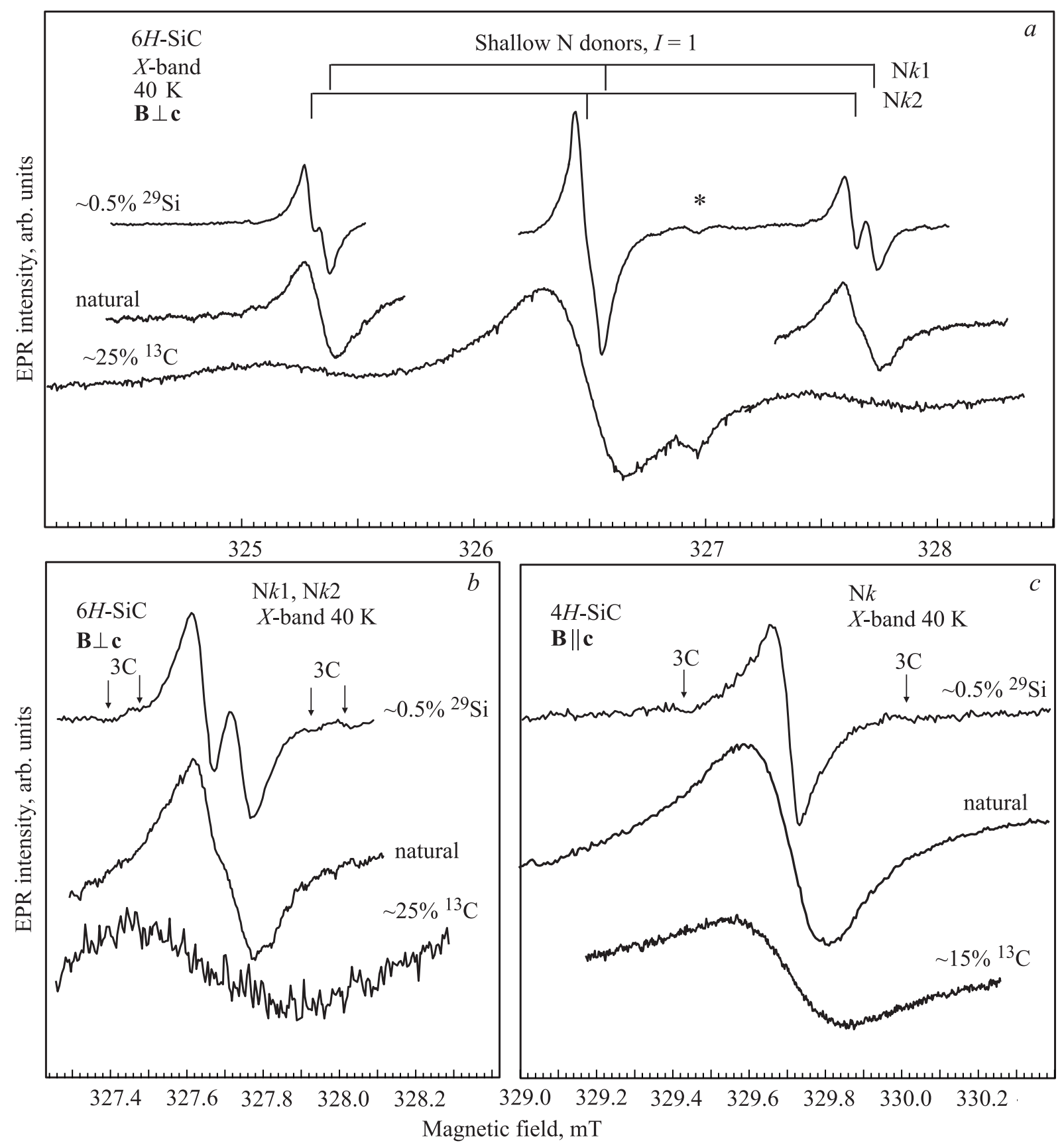

Рис. 5. а) Спектры ЭПР мелких доноров азота в трех кристаллах $6 H$-SiC: с природным содержанием изотопов; обогащенном изотопом ${ }^{28} \mathrm{Si}\left(<0.5 \%{ }^{29} \mathrm{Si}\right)$; обогащенном изотопом ${ }^{13} \mathrm{C}(\sim 25 \%)$. Спектры зарегистрированы при температуре $40 \mathrm{~K}$ в ориентации магнитного поля $\mathbf{B} \perp \mathbf{c}$. Реперный сигнал от кварца, обозначен звездочкой. $b)$ Высокополевые компоненты спектров ЭПР мелких доноров азота в $6 H$-SiC в $k 1$-, $k 2$-позициях, в ориентации $\mathbf{B} \perp \mathbf{c}$. Для кристалла с малым содержанием изотопа ${ }^{29} \mathrm{Si}$ стрелками показаны сателлиты для сверхтонкого взаимодействия с тремя атомами углерода. $c$ ) Высокополевые компоненты спектров ЭПР мелких доноров азота в $4 H-\mathrm{SiC}$ в $k$-позиции в ориентации В $\|$ с. Для кристалла с малым содержанием изотопа ${ }^{29} \mathrm{Si}$ стрелками показаны сателлиты для сверхтонкого взаимодействия с тремя атомами углерода.

Видно, что уменьшение концентрации изотопа ${ }^{29} \mathrm{Si}$ на порядок приводит к существенному сужению линий ЭПР. Однако обогащение ${ }^{13} \mathrm{C}$ не привело к заметному уширению линии ЭПР. Из этого следует, что изменение ширины линии ЭПР в кристаллах с модифицированным изотопным составом в $4 \mathrm{H}$ - $\mathrm{SiC}$ заметно отличается от то- го, что наблюдалось в $6 H-\mathrm{SiC}$. В кристалле, обедненном ${ }^{29} \mathrm{Si}$, обнаружены две дополнительные линии (обозначенные стрелками), расположенные симметрично относительно центральной линии. Подобные сателлиты наблюдались и для других линий $\mathrm{N} k$. Поскольку эти линии были видны в кристаллах с низким содержанием ${ }^{29} \mathrm{Si}$, как 


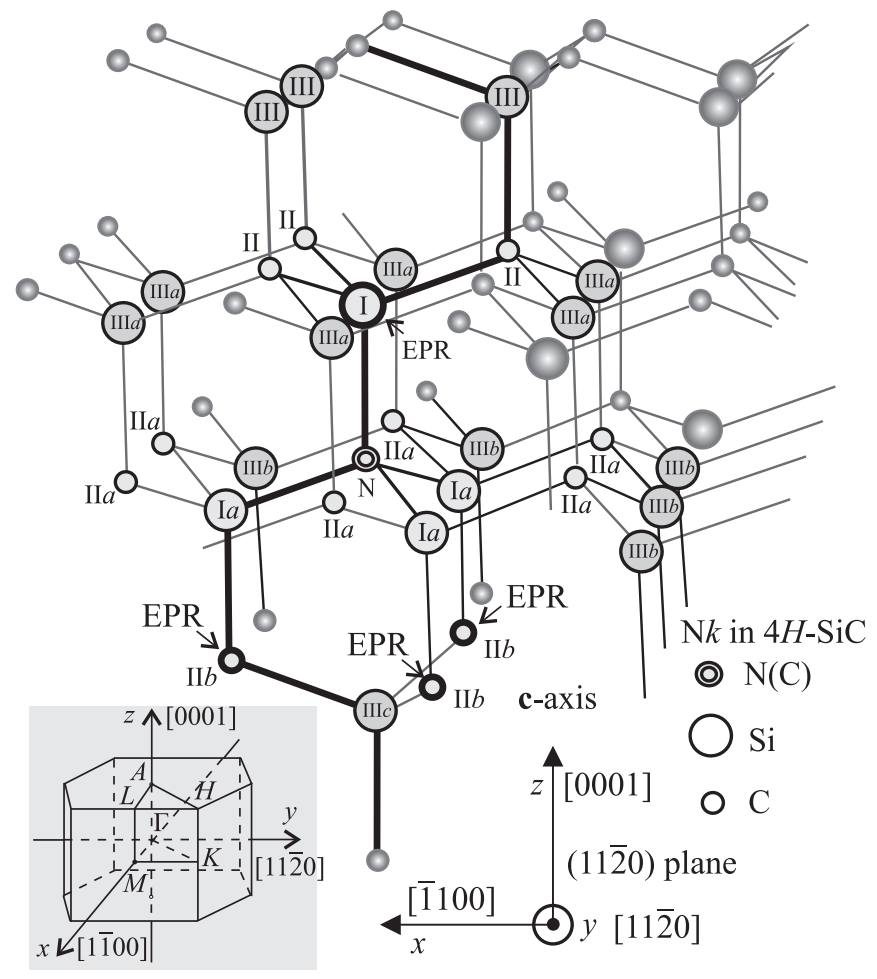

Рис. 6. Схема кристаллической структуры вокруг позиции $k$ азота $(\mathrm{N} k)$ в $4 H$-SiC. Показано направление с-оси, ступенчатая структура нарисована жирными линиями. Внизу указана ориентация осей $x, y, z$. Плоскость $\{11 \overline{2} 0\}$ содержит оси $\mathbf{c}(z)$, и $x$ и совпадает с поверхностью страницы. Указан атом $\mathrm{N}$ в позиции С. Римские цифры, помещенные вблизи атомов $\mathrm{Si}$ и $\mathrm{C}$, соответствуют номерам, обозначающим группы, относящиеся к одинаковым координационным сферам. Окружение позиции $k 1$ в $6 H$-SiC является точно таким же, как и окружение позиции $k$ в $4 H$-SiC. Стрелками показаны один атом кремния и три атома углерода (окружности выделены толстыми линиями), для которых сверхтонкие взаимодействия разрешены в спектрах ЭПР и которые нужно исключить из анализа данных по ДЭЯР [45], вносящих вклад в ширины линий ЭПР. На вставке обратная решетка $4 H-\mathrm{SiC}$ и $6 H-\mathrm{SiC}$ с параметрами решетки $4 \pi /(\sqrt{ } 3 a)$ (расстояние от центра шестиугольника к углу) и $2 \pi / c$ (высота шестиугольника). Решетка поворачивается на $60^{\circ}$ вокруг с-оси относительно решетки в реальном пространстве. Показаны положения особых точек симметрии вместе с ориентацией шестиугольника относительно кристаллографической системы координат, $x, y$ и $z$ в реальном пространстве. Точка Г является центром шестиугольника. Точка $M$ лежит в плоскости (112̄0), точка $K$ в плоскости $(\overline{1} 100)$ и точка $L$ лежит выше точки $M$ вдоль направления [0001].

и в случае с $6 H$-SiC, они обусловлены CТ-взаимодействием с ${ }^{13} \mathrm{C}$; для кристаллов с природным содержанием изотопа ${ }^{13} \mathrm{C}$ сателлиты маскируются более широкими линиями доноров азота. Как и в случае с кристаллом $6 \mathrm{H}$-SiC, отношение интенсивностей позволяет предположить, что сателлиты возникают от СТ-взаимодействия с тремя атомами углерода, с константой СТ-взаимодействия $0.6 \mathrm{mT}(16.8 \mathrm{MHz})$. Как видно из рис. 5, $b$, фор- ма линий ЭПР для кристалла с природным изотопным составом отличается от полученной формы линий в кристалле, обогащенном ${ }^{13} \mathrm{C}$. Действительно, крылья линии ЭПР в последнем случае спадают существенно медленнее. Наблюдаемая форма линии отражает наличие сателлитов, интенсивность которых значительно выше, в кристаллах, обогащенных изотопом ${ }^{13} \mathrm{C}$. В кристаллах $6 H$-SiC с природным изотопным составом наблюдаются сателлиты, соответствующие практически изотропному СТ-взаимодействию с одним атомом $\mathrm{Si}$, с константой $0.96 \mathrm{mT}(26.9 \mathrm{MHz})$. Такие сателлиты не обнаружены в кристаллах с низким содержанием ${ }^{29} \mathrm{Si}$. В кристаллах $4 H$-SiC взаимодействие с одним атомом кремния равно $1.46 \mathrm{mT}$ (41 MHz).

Таким образом, в спектрах ЭПР мелких доноров азота, занимающих $k$-позиции в политипах $4 H-\mathrm{SiC}$ и $6 \mathrm{H}$-SiC и характеризующихся самыми глубокими уровнями (табл. 1), наряду с неразрешенной СТ-структурой, определяющей ширины линий ЭПР, имеются СТ-взаимодействия с ближайшими координационными сферами. Эти СТ-взаимодействия могут быть разрешены в виде сателлитов в спектрах ЭПР (рис. 5, $b$ и $c$ ). Сателлиты, обусловленные СТ-взаимодействием с кремнием, наблюдались в $[44,45]$, и сателлиты, обусловленные взаимодействием с углеродом, были зарегистрированы в работе [46]. Однозначная идентификация этих дополнительных линий ЭПР проведена с помощью исследования кристаллов с измененным изотопным составом (что позволило наблюдать взаимодействия с углеродом благодаря сужению линий ЭПР [46]) и методом ДЭЯР [55]. На рис. 6 выделены атомы кремния и углерода, СТ-взаимодействие с которыми разрешено в спектрах ЭПР доноров азота.

2.3. Обсуждение результатов. Донорный атом азота, будучи элементом V группы, имеет пять валентных электронов, четыре из которых образуют валентные связи с четырьмя ближайшими атомами решетки, а пятый находится в кулоновском поле остающегося положительного заряда. В приближении теории эффективной массы (ЭМ) [44,45] слабо связанный электрон рассматривается как водородоподобный атом, в котором кулоновское притяжение ядра донора уменьшено из-за диэлектрической проницаемости полупроводника $\varepsilon$. При этом считается, что электрон движется по своей орбите с эффективной массой электрона в зоне проводимости. Тогда волновая функция локализованного донорного электрона записывается в виде произведения решения уравнения Шредингера для водородоподобного атома, образованного донором и слабо связанным электроном, и функции Блоха для электрона в зоне проводимости. Другими словами, функция Блоха, описывающая электроны зоны проводимости, модулируется огибающей функцией, которая является решением соответствующего водородоподобного уравнения Шредингера. Результатом является формирование связанного донорного состояния с энергией ионизации порядка десятков $\mathrm{meV}$. 
Карбид кремния, подобно кремнию, относится к IV группе Периодической таблицы элементов, и поэтому наряду с существенными различиями обладает качественно подобной структурой энергетических уровней. Эти кристаллы являются непрямозонными полупроводниками, поскольку зона проводимости имеет несколько минимумов, смещенных относительно центра зоны Бриллюэна. Характер этого сдвига зависит от природы полупроводникового материала. Кремний имеет шесть минимумов, смещенных в направлениях [100], в $\mathrm{SiC}$ вид зоны проводимости зависит от политипа. Вследствие многодолинного характера зоны проводимости уровни мелких доноров, которые можно рассматривать как отщепленные от зоны проводимости, являются вырожденными со степенью вырождения, равной числу минимумов, например шести в кремнии, которые описываются состояниями $A_{1}, E$ и $T_{2}$. Из-за нарушения теории эффективной массы вблизи донорной примеси вырождение снимается. Причиной расщепления уровня, названного долинно-орбитальным расщеплением (см. табл. 1), является различие в распределении электронов в состояниях $A_{1}, E$ и $T_{2}$ вблизи донорной примеси. Наиболее сильное отличие наблюдается в энергии для синглетного состояния $A_{1}$, так как волновая функция в этом случае имеет максимальную амплитуду на донорном атоме.

Волновая функция донорного электрона (например, для кремния и кубического политипа $3 C$ - $\mathrm{SiC}$ ) записывается

$$
\psi(\mathbf{r})=\sum_{j=1}^{6} \alpha_{j} F_{j}(\mathbf{r}) \varphi_{j}(\mathbf{r}),
$$

где $\varphi_{j}(\mathbf{r})=u_{j}(\mathbf{r}) e^{i \mathbf{k}_{j} \mathbf{r}}-$ функция Блоха в $j$-м минимуме, который локализуется в точке $\mathbf{k}_{j}$, и $u_{j}(\mathbf{r})-$ периодическая функция. Коэффициенты $\alpha_{j}$ характеризуют относительный вклад каждой долины и, следовательно, образуют различные комбинации волновых функций, описывающих область вблизи каждого минимума в зоне проводимости. Поскольку a priori форма основного состояния неясна, наличие СТ-взаимодействия на донорном атоме, как правило, дает основание утверждать, что основным состоянием является синглет $A_{1}$, так как только в этом состоянии имеется конечная плотность волновой функции на ядре донора. В этом случае волновая функция полностью симметрична и электрон равномерно распределен по всем долинам, т.е. для $\mathrm{Si}$ или $3 C-\mathrm{SiC}$ волновая функция имеет вид

$$
\psi(\mathbf{r})=\frac{1}{\sqrt{6}} \sum_{j=1}^{6} F_{j}(\mathbf{r}) u_{j}(\mathbf{r}) e^{i \mathbf{k}_{j} \mathbf{r}}
$$

B $\mathrm{SiC}$ степень вырождения и дальнейшее расщепление зависят от политипа. В кубическом политипе $3 C$ - $\mathrm{SiC}$ ситуация аналогична кремнию, т.е. минимумы зоны проводимости расположены вдоль осей $\langle 100\rangle$ с поверхностями постоянной энергии вблизи минимумов в виде эллипсоидов, вытянутых вдоль направлений $\langle 100\rangle$. Эффективные массы вдоль и перпендикулярно направлению оси эллипсоида равны $m_{l}=0.677 m_{0}$ и $m_{t}=0.247 m_{0}$ соответственно, где $m_{0}$ - масса свободного электрона. В гексагональных политипах наблюдается более сложная картина. Имеется существенное различие в положении минимумов зоны проводимости для политипов $4 H-\mathrm{SiC}$ и $6 H-\mathrm{SiC}$, что в конечном итоге и приводит к различию их электронных свойств.

Для политипа $4 H-\mathrm{SiC}$ минимум зоны проводимости находится в точке $M$, тогда как для политипа $6 H-\mathrm{SiC}$ положение минимума локализуется между точками $M$ и $L$ на расстоянии примерно $60 \%$ от точки $M$ (см. вставку на рис. 6). Особенности зонной структуры приводят к тому, что для обоих политипов эффективные массы в плоскости, перпендикулярной оси с, примерно одинаковы $\left(m_{\perp}=0.445 m_{0}\right.$ для $4 H-\mathrm{SiC}$ и $m_{\perp}=0.43 m_{0}$ для $6 H$-SiC), но существенно отличаются вдоль оси с. Так, для $4 H-\mathrm{SiC}$ эффективная масса вдоль оси с $m_{\|}=0.32 m_{0}$, а для $6 \mathrm{H}-\mathrm{SiC} m_{\|}=1.7 m_{0}$. Таким образом, для политипа $4 H-\mathrm{SiC}$ (и $3 \mathrm{C}-\mathrm{SiC}$ ) электронная волновая функция почти сферически симметрична, тогда как для $6 \mathrm{H}-\mathrm{SiC}$ электронная волновая функция существенно сжата вдоль оси с, т. е. имеет форму „блина“.

Целесообразно использовать усредненные эффективные массы $m^{*}$ для электронов в зоне проводимости. „Эффективный боровский радиус“ волновой функции мелкого донора дается выражением $a^{*}=\left[\varepsilon /\left(m^{*} / m_{0}\right)\right] a_{0}$, где $a_{0}=0.529 \AA-$ боровский радиус. Выражение для энергии ионизации мелкого донора имеет вид $E_{\text {eff }}=\left[\left(m^{*} / m_{0}\right) / \varepsilon^{2}\right] E_{0}, E_{0}$ - энергия Ридберга. Таким образом, „эффективный боровский радиус“ для электронной волновой функции составляет $a^{*}=15 \AA$ в $3 C-\mathrm{SiC}, a^{*}=13 \AA \quad$ в $4 H-\mathrm{SiC}$ и $a^{*}=7.2 \AA \quad$ в $6 H-\mathrm{SiC}$, соответствующие энергии ионизации $E_{\text {eff }}$ для мелкого донора $-47 \mathrm{meV}$ в $3 C-\mathrm{SiC}, 54 \mathrm{meV}$ в $4 H-\mathrm{SiC}$ и $101 \mathrm{meV}$ в $6 \mathrm{H}-\mathrm{SiC}$.

Следует отметить, что речь идет о волновой функции донорного электрона, который не локализован в какойто специфической позиции $k$ или $h$ рассматриваемого политипа. Однако вполне естественно, что свойства донорных электронов будут нести информацию о волновой функции свободного электрона. Таким образом, существенное отличие в свойствах волновых функций на атомах $\mathrm{N}$ для $k$ - или $h$-позиций, которое не зависит от политипа, обусловлено, по-видимому, только локальной симметрией этих позиций и не отражает общую волновую функцию зонного электрона. Эти свойства должны быть отражены в СТ-взаимодействиях с различными координационными сферами атомов С и $\mathrm{Si}$, которые пропорциональны плотности донорной волновой функции (4) $\left|\psi\left(\mathbf{r}_{l}\right)\right|^{2}$ на ядре атома в узле $l$. При оценках величины плотности волновой функции на отдельном ядре используют безразмерную величину, называемую коэффициентом усиления, который характеризует степень локализации волновой функции вблизи ядра, т.е. является отношением плотности фактической волновой 
функции на ядре к плотности огибающей волновой функции в том же месте. Коэффициент усиления для областей, где огибающая волновая функция изменяется медленно (в соответствии с теорией ЭМ и без учета интерференционных эффектов), может быть выражен в виде безразмерной величины $\eta \equiv\left|u_{j}\left(\mathbf{r}_{l}\right)\right|^{2} /\left\langle u_{j}(\mathbf{r})\right\rangle^{2}$, где знаменатель содержит усредненную величину функции Блоха по элементарной ячейке. Эта величина не зависит от положения атома в решетке, но зависит от типа атома $\mathrm{Si}$ или $\mathrm{C}$.

Из табл. 1 видно, что энергии донорных уровней для $3 C$-SiC и для гексагональных позиций соответствуют энергиям, рассчитанным по теории ЭМ, тогда как уровни для $k$-позиций существенно превышают эти величины. Тот факт, что СТ-структура мала в $\mathrm{SiC}$ для мелких донорных уровней в $3 C-\mathrm{SiC}$ и в гексагональных позициях, вероятно, свидетельствует о том, что распределение волновых функций донорных электронов в этих случаях существенно отличается от соответствующего распределения для доноров в квазикубических позициях и, следовательно, СТ-структура в этом случае в спектрах ЭПР практически не разрешена.

Имеются три основных эффекта, определяющие СТ-взаимодействия для мелких доноров в кристаллах IV группы, являющихся непрямозонными полупроводниками: $(i)$ Плавно уменьшающаяся модуляция СТ-взаимодействий с увеличением расстояния от донора [в рамках теории ЭМ описывается огибающей функцией $F(\mathbf{r})]$; (ii) Безразмерные коэффициенты усиления, описывающие локализацию спиновой плотности на атомах и зависящие от типа атома и кристалла; и (iii) Интерференционные эффекты, обусловленные наличием нескольких долин у этих полупроводников, приводящие к нарушению описываемой функцией $F(\mathbf{r})$ монотонности спада СТ-взаимодействий с расстоянием на отдельных атомах или группах атомов. Отметим, что эффект интерференции затрудняет интерпретацию спектров ЭПР и ДЭЯР в непрямозонных полупроводниках в отличие от прямозонных ( $\mathrm{ZnO}, \mathrm{AgCl}, \mathrm{AgBr})$, в которых СТ-взаимодействия с лигандными атомами были сравнительно надежно интерпретированы.

Окружающие ионы мелкого донора $\mathrm{N} k$ в $4 H-\mathrm{SiC}$ (окружение узла $k 1$ в $6 H-\mathrm{SiC}$ точно такое же, как и окружение этого $k$-узла в $4 H-\mathrm{SiC})$, для которых CТ-взаимодействия разрешены методами ЭПР и ДЭЯР и проанализированы в данном разделе, представлены на pис. 6, на котором ссхематически показана кристаллическая структура вокруг позиции $k$ азота $(\mathrm{N} k)$ в $4 H-\mathrm{SiC}$. Показано направление с-оси, ступенчатая структура нарисована жирными линиями. Внизу указана ориентация осей $x, y, z$. Плоскость $\{11 \overline{2} 0\}$ содержит оси $\mathbf{c}(z)$ и $x$ и совпадает с поверхностью страницы. Указан атом $\mathrm{N}$ в позиции С. Римские цифры, помещенные вблизи атомов $\mathrm{Si}$ и $\mathrm{C}$, соответствуют номерам, обозначающим группы, относящиеся к одинаковым координационным сферам. Окружение позиции $k 1$ в $6 H$-SiC является точно таким же, как и окружение позиции $k$ в $4 H-\mathrm{SiC}$.
Стрелками показаны один атом кремния и три атома углерода (окружности выделены жирными линиями), для которых СТ-взаимодействия разрешены в спектрах ЭПР и которые нужно исключить из анализа данных по ДЭЯР [46], вносящих вклад в ширины линий ЭПР.

Мы будем рассматривать только координационные сферы, образованные атомами $\mathrm{Si}$ и $\mathrm{C}$ и обозначенные I, I $a$, II, II $a$, III, III $a$, III $b$ и III $c$. СТ-структура первой координационной сферы (один атом $\mathrm{Si}$, обозначен I) и трех атомов углерода второй координационной сферы (обозначены II $b$ ) была разрешена в спектрах ЭПР. СТ-структура и плотность неспаренного электрона вне этих координационных сфер были проанализированы в работе [46]. Почти изотропное СТ-расщепление, вызванное взаимодействием с одним атомом $\mathrm{Si}$ в первой координационной сфере, обозначенное I на рис. 6, составляет: $1.46 \mathrm{mT}(41 \mathrm{MHz})$ в $4 H-\mathrm{SiC}$ и $0.96 \mathrm{mT}(26.9 \mathrm{MHz})$ в $6 \mathrm{H}$-SiC соответственно.

Изотропное СТ-расщепление с тремя атомами углерода, обозначенными на рис. 6 как II $b$, которые связаны вдоль с-оси с ядрами трех атомов кремния, обозначенными $\mathrm{I} a$, составляет $0.6 \mathrm{mT}(16.8 \mathrm{MHz})$ для $4 H-\mathrm{SiC}$ и $0.5 \mathrm{mT}(14 \mathrm{MHz})$ для $6 \mathrm{H}-\mathrm{SiC}$. Наблюдаемые изотропные СТ-взаимодействия дают возможность оценить плотность неспаренного электрона на $s$-орбиталях $\mathrm{Si}$ и С и тем самым рассчитать степень локализации электрона на этих орбиталях. Соответствующие степени локализации неспаренного электрона (спиновой плотности) на одном атоме $\mathrm{Si}$ в $4 H-\mathrm{SiC}$ и $6 H-\mathrm{SiC}$ соответственно составляют 0.89 и $0.6 \%$, а на каждом из трех атомов С в $4 H-\mathrm{SiC}$ и $6 H-\mathrm{SiC}$ эти величины равны 0.44 и $0.37 \%$ соответственно. Важно отметить, что отношение спиновых плотностей на одном атоме $\mathrm{Si}$ для политипов $4 H-\mathrm{SiC}$ и $6 H$-SiC, равное 1.5 , в пределах экспериментальной ошибки совпадает с соответствующим отношением спиновых плотностей на атоме азота (табл. 1). Это свидетельствует о том, что структура центров $\mathrm{N}$ в $k$-позициях в $4 H-\mathrm{SiC}$ и $6 H-\mathrm{SiC}$ является одинаковой и один атом $\mathrm{Si}$ находится в ближайшей позиции к атому $\mathrm{N}$, при этом плотность волновой функции при переходе от $\mathrm{N}$ к $\mathrm{Si}$ плавно уменьшается примерно в 3 раза. Отношение спиновых плотностей на каждом из атомов С для политипов $4 H-\mathrm{SiC}$ и $6 H-\mathrm{SiC}$, равное 1.2 , уже значительно меньше, чем соответствующее отношение спиновых плотностей на атоме $\mathrm{N}$, т.е. эти атомы занимают более удаленные позиции по отношению к азоту. Таким образом, результаты настоящей работы подтверждают, что азот в $k$-позициях замещает С. Можно предположить, что локализация на оставшихся трех ближайших атомах $\mathrm{Si}$ (I $a$ на рис. 6) того же порядка, что и на одном атоме $\mathrm{Si}$, для которого наблюдалось изотропное СТ-расщепление в спектре ЭПР. При этом весьма вероятно, что локализация будет осуществляться на $s$ - и $p$-орбиталях, т.е. CТ-взаимодействие с тремя атомами Si первой координационной сферы может быть анизотропным и поэтому не разрешается в спектрах ЭПР, поскольку даже при незначительном перераспределении неспаренного электрона 
на $p$-орбиталь изотропная константа СТ-взаимодействия сильно уменьшится. Анизотропное СТ-взаимодействие с тремя атомами кремния в базисной плоскости, которые обозначены І $a$, из данных ДЭЯР составляет: $a=6.54 \mathrm{MHz}, b=1.26 \mathrm{MHz}, b^{\prime}=0.75 \mathrm{MHz}, s=0.14 \%$, $p=1.1 \%$. Таким образом, в $4 H-\mathrm{SiC}$ суммарная локализация неспаренного электрона на центральном атоме $\mathrm{N}$ и первой сфере (из четырех атомов $\mathrm{Si}$ I и І $a$, рис. 6) будет составлять 7.4\%. К этому следует добавить $2.2 \%$, которые приходятся на предполагаемые три атома С второй сферы. Таким образом, в сумме в пределах первых двух координационных сфер спиновая плотность составляет примерно $10 \%$. Для $6 H$-SiC соответствующие количества будут в 1.5 раза меньше.

Эти величины следует добавить к тем, которые были получены в результате исследований методом ДЭЯР в работе [46], и которые соответствуют СТ-взаимодействиям для $k$-позиций азота, разрешенным в спектрах ЭПР. Поскольку ширины линий ЭПР отражают распределение плотности неспаренного электрона мелкого донора для более удаленных координационных сфер в случае $k$-позиций в $\mathrm{SiC}$ (вероятно, начиная с третьей координационной сферы), для которых выполняется приближение теории ЭМ, рассмотрим СТ-взаимодействия с этими координационными сферами более детально. $\mathrm{B} 4 \mathrm{H}-\mathrm{SiC}$, согласно исследованиям ДЭЯР, существуют сильные СТ-взаимодействия с атомами С в двух группах: с $a=5.02 \mathrm{MHz}(s=0.13 \%)$ и с $a=4.4 \mathrm{MHz}$ и $b=0.23 \mathrm{MHz}(s=0.12 \%$ и $p=0.22 \%)$. Эти СТ-взаимодействия могут быть отнесены к атомам C, обозначенным II (3 атома) и II $a$ (6 атомов) на рис. 6. $\mathrm{B} 4 \mathrm{H}-\mathrm{SiC}$, согласно исследованиям ДЭЯР, сильнейшие СТ-взаимодействия с атомами $\mathrm{Si}$ в двух группах: с $a=4.8 \mathrm{MHz}(s=0.1 \%), b=0.04 \mathrm{MHz}(p=0.04 \%)$; и с $a=4.14 \mathrm{MHz}$ и $b=0.34 \mathrm{MHz}(s=0.09 \%$ и $p=0.3 \%)$ со связями вдоль с-оси можно отнести к атомам $\mathrm{Si}$, обозначенным III и III $c$ на рис. 6. Для групп $\mathrm{Si}$ III $a$ и III $b$ (рис. 6), расположенных в базисной плоскости, СТ-взаимодействия являются следующими: $a=2.3 \mathrm{MHz}$ $(s=0.05 \%), b=0.31 \mathrm{MHz}(p=0.27 \%)$ и $a=2.0 \mathrm{MHz}$ $(s=0.04 \%), b=0.12 \mathrm{MHz}(p=0.11 \%)$.

Аналогичным образом данные ДЭЯР [46] для 6H-SiC можно отнести к различным координационным сферам $\mathrm{Si}$ и C, представленным на рис. 6, так как окружение $k 1$-узла в $6 H$-SiC аналогично окружению $k$-узла в $4 H-\mathrm{SiC}$.

Поскольку ширины линий ЭПР отражают распределение плотности неспаренного электрона мелкого донора по координационным сферам в случае $k$-позиции $\mathrm{N}$ в $\mathrm{SiC}$ (за исключением сильных СТ-взаимодействий, которые были разрешены в спектрах ЭПР), к которой применимо приближение ЭМ, мы рассмотрим СТ-взаимодействия с этими координационными сферами более подробно.

Формула для ширины на полувысоте неразрешенной линии ЭПР $\Delta B$ для случая, в котором данная ширина определяется сверхтонкими взаимодействиями имеет вид

$$
\begin{aligned}
\Delta B^{2}= & 2\left(\sum_{i}^{R} f(\mathrm{Si}) n_{i}(\mathrm{Si})\left(\frac{a_{i}(\mathrm{Si})}{2}\right)^{2}\right. \\
& \left.+\sum_{j}^{M} f(\mathrm{C}) n_{j}(\mathrm{C})\left(\frac{a_{j}(\mathrm{C})}{2}\right)^{2}\right),
\end{aligned}
$$

где $n_{i}$ и $n_{j}$ - количество эквивалентных узлов в $i$-й координационной сфере $\mathrm{Si}$ и $j$-й координационной сфере С. $a_{i}$ и $a_{j}-$ сверхтонкие взаимодействия для эквивалентных узлов в $i$-й координационной сфере $\mathrm{Si}$ и $j$-й координационной сфере $\mathrm{C} . f(\mathrm{Si})$ и $f(\mathrm{C})$ - относительная распространенность ${ }^{29} \mathrm{Si}$ и ${ }^{13} \mathrm{C}(f(\mathrm{Si})=0.047$ и $f(\mathrm{C})=0.011$ в природных образцах $). f(\mathrm{Si}) n_{i}(\mathrm{Si})$ и $f(\mathrm{C}) n_{j}(\mathrm{C})$ - вероятности обнаружить $i$-й и $j$-й узел решетки, занимаемый ${ }^{29} \mathrm{Si}$ или ${ }^{13} \mathrm{C}$ соответственно. Сложение распространяется по сферам кремния $R$ и сферам углерода $M$, которые вносят вклад в ширину линии ЭПР. Интерпретация данных ДЭЯР по ${ }^{13} \mathrm{C}$ и ${ }^{29} \mathrm{Si}$ и расчет ширины линии ЭПР представлены в работе [46]. Обсуждалась ширина линии донора $\mathrm{N} k$ в кристалле 4H-SiC, поскольку спектры ДЭЯР более информативны для этого центра, чем для доноров $\mathrm{N}$ в $6 \mathrm{H}-\mathrm{SiC}$. УГлы Эйлера сверхтонких тензоров дают информацию о координационных сферах, к которым они принадлежат. К тому же, вероятно, можно заметить замечательное сходство между донором $\mathrm{N} k$ в $4 H-\mathrm{SiC}$, с одной стороны, и мелкими донорами $\mathrm{P}(\mathrm{As}, \mathrm{Sb})$ в $\mathrm{Si}$, с другой стороны, когда сравниваются СТ-взаимодействия с ядром примеси и окружающими ядрами ${ }^{29} \mathrm{Si}$. На основании этого сходства в работе [46] предложена идентификация ряда линий ДЭЯР для ядер ${ }^{29} \mathrm{Si}$, окружающих донор $\mathrm{N} k$. СТ-взаимодействие с центральным атомом примеси приводит в обоих случаях к $s$-подобной спиновой плотности и тому, что это взаимодействие имеет тот же порядок величины, что и ближайшие соседние атомы. Три ближайших атома кремния, соседних к атому $\mathrm{N}$ (в положении углерода) в плоскости, перпендикулярной с-оси, показывают удивительно большое анизотропное СТ-взаимодействие, которое почти того же размера, что и изотропное СТ-взаимодействие и похоже на сферу с симметрией (111) для донора $\mathrm{P}$ в кремнии. При расчете были включены только те координационные сферы, которые имеют изотропное СТ-взаимодействие, которое больше, чем $1 \mathrm{MHz}$. Авторы [46] использовали число шесть для количества атомов в каждой координационной сфере. В случае кристалла, обогащенного изотопом ${ }^{13} \mathrm{C}$, число ядер ${ }^{13} \mathrm{C}$, с которыми взаимодействует донорный электрон, значительно возрастает, и, следовательно, ширина линии увеличивается. Результаты расчета, а также экспериментальные значения,

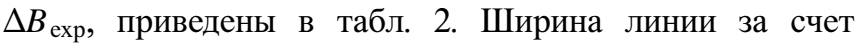
атомов углерода, $\Delta B(\mathrm{C})$, и общая ширина линии, $\Delta B_{\text {total, }}$ даются для необогащенного (природного), а также для обогащенного случаев. Понятно, что расчеты хорошо согласуются с экспериментально полученной шириной 
Таблица 3. Экспериментальные и рассчитанные ширины линий ЭПР в $\mathrm{mT}$ для мелких доноров азота в $k$-позиции в $4 H$-SiC и в $k 1$-позиции в $6 H$ - $\mathrm{SiC}$ с различным содержанием изотопов ${ }^{13} \mathrm{C}$ и ${ }^{29} \mathrm{Si}$ : природном, обогащенном изотопом ${ }^{13} \mathrm{C}(25 \%$ для $6 H$ - $\mathrm{SiC}$ и $15 \%$ для $4 H-\mathrm{SiC})$, с низким содержанием ${ }^{29} \mathrm{Si}(0.5 \%)$. В каждом образце изменялось содержание только одного изотопа

\begin{tabular}{c|c|c|c|c|c|c}
\hline \multirow{2}{*}{ Политип } & \multicolumn{3}{|c|}{ Эксперимент } & \multicolumn{3}{c}{ Расчет } \\
\cline { 2 - 7 } & $\Delta B$ (природный $)$ & $\Delta B(\mathrm{C}-13)$ & $\Delta B(\mathrm{Si}-29)$ & $\Delta B$ (природный $)$ & $\Delta B(\mathrm{C}-13)$ & $\Delta B(\mathrm{Si}-29)$ \\
\hline $4 H-\mathrm{SiC}$ & 0.21 & 0.35 & 0.07 & 0.21 & 0.29 & 0.08 \\
$6 H-\mathrm{SiC}$ & 0.13 & 0.5 & 0.12 & 0.12 & 0.55 & 0.11
\end{tabular}

линии и, таким образом, можно сделать вывод о том, что мы не пропустили большие СТ-взаимодействия с соседними атомами ${ }^{29} \mathrm{Si}$ или ${ }^{13} \mathrm{C}$, за исключением тех, для которых разрешенная СТ-структура наблюдалась в спектрах ЭПР.

Согласно формуле (5), ширина линии ЭПР для мелких доноров $\mathrm{N}$ в $\mathrm{SiC}$ имеет сложную структуру из-за наличия двух типов атомов, $\mathrm{Si}$ и С. Фактическое значение ширины линии зависит от вклада СТ-взаимодействия с ядрами ${ }^{29} \mathrm{Si}$ и ${ }^{13} \mathrm{C}$ в ширину линии. Предполагая, что ширина линии полностью определяется СТ-взаимодействием с ${ }^{13} \mathrm{C}$, отношение ширины линии для $6 \mathrm{H}-\mathrm{SiC}$, обогащенного ${ }^{13} \mathrm{C}$ до $25 \%$, к ширине линии кристалла с природным изотопным составом рассчитывается как $(0.25 / 0.011)^{1 / 2}=4.8$ и ширина линии ЭПР должна быть $0.62 \mathrm{mT}$ (на основе экспериментальной ширины линии в природном кристалле). Аналогичное соотношение для $4 H-\mathrm{SiC}$, обогащенного ${ }^{13} \mathrm{C}$ до $15 \%$, будет $(0.15 / 0.011)^{1 / 2}=3.7$, а ширина линии ЭПР составит $0.78 \mathrm{mT}$. Если теперь предположить, что ширина линии полностью определяется СТ-взаимодействием с ${ }^{29} \mathrm{Si}$, вычисленное отношение ширины линии для $6 H-\mathrm{SiC}$ с содержанием $0.5 \%{ }^{29} \mathrm{Si}$ (кристалл обогащен ${ }^{28} \mathrm{Si}$ ) к кристаллу с природным изотопным составом дает $(0.005 / 0.047)^{1 / 2}=0.33$; так что ширина линии ЭПР должна быть $0.04 \mathrm{mT}$. Аналогичным образом, ширина линии ЭПР для $4 H-\mathrm{SiC}$ может быть найдена $0.07 \mathrm{mT}$.

Сравнение этих величин с наблюдаемыми в эксперименте в $X$-диапазоне (табл. 3) показывает, что первый случай (в котором ширина линии полностью определяется СТ-взаимодействием с ${ }^{13} \mathrm{C}$ ) практически реализуется для политипа $6 H-\mathrm{SiC}$, тогда как для $4 H-\mathrm{SiC}$ реализуется второй случай, т.е. ширина линии главным образом определяется СТ-взаимодействием с ${ }^{29} \mathrm{Si}$. Для политипа $6 \mathrm{H}$-SiC увеличение содержания изотопа ${ }^{13} \mathrm{C}$ примерно в 20 раз приводит к уширению линии ЭПР почти в 4 раза, тогда как уменьшение содержания изотопа ${ }^{29} \mathrm{Si}$ более чем в 10 раз практически не изменяет ширину линии. Напротив, в $4 H-\mathrm{SiC}$ аналогичное уменьшение содержания изотопа ${ }^{29} \mathrm{Si}$ привело к сужению линии ЭПР в 3 раза, при этом значительное увеличение содержания ${ }^{13} \mathrm{C}$ не привело к существенному увеличению ширины линии. Таким образом, на основании представленных исследований ЭПР можно сделать вывод, что имеется существенное различие в распределении волновых функ- ций неспаренного электрона донора азота для политипов $4 H-\mathrm{SiC}$ и $6 H$-SiC. В $4 H-\mathrm{SiC}$ волновая функция главным образом локализована на атомах кремния, тогда как в случае $6 H-\mathrm{SiC}$ волновая функция локализована в основном на атомах углерода. Этот вывод находится в соответствии с результатами исследований методом ДЭЯР [46], в которых также было обнаружено существенное различие в пространственном распределении волновой функции мелких доноров $\mathrm{N}$ в политипах $4 H-\mathrm{SiC}$ и $6 H$-SiC. Было показано, что для координационных сфер, взаимодействия с которыми определяют ширины линий ЭПР, неспаренный электрон в 4H-SiC преимушественно локализуется на атомах кремния, а в $6 \mathrm{H}$-SiC - на атомах углерода. При этом на кремнии неспаренный электрон распределен примерно одинаково на $s$ - и p-орбиталях, тогда как на углероде неспаренный электрон распределен в основном на $s$-орбиталях. Иными словами, в указанных политипах коэффициенты усиления для $\mathrm{Si}$ и С существенно различаются. Так, если судить по максимальным изотропным константам СТ-взаимодействия с ${ }^{13} \mathrm{C}$, равным в $4 H-\mathrm{SiC}$ и $6 H-\mathrm{SiC}$ 5.02 и $10.75 \mathrm{MHz}(k 2)$ соответственно [46], коэффициент усиления для углерода в $6 H-\mathrm{SiC}$ примерно в 2 раза больше соответствующей величины в $4 H$-SiC. В то же время для распределения неспаренного электрона на $s$-орбиталях кремния наблюдается обратная картина: 6.54 и $3.86 \mathrm{MHz}$ в $4 H-\mathrm{SiC}$ и $6 H-\mathrm{SiC}$ соответственно. Это означает, что коэффициент усиления для изотропного распределения спиновой плотности на кремнии в 1.7 раза больше в $4 H-\mathrm{SiC}$ по сравнению с $6 H$-SiC. При этом следует учесть, что аналогичный коэффициент усиления для распределения неспаренного электрона на p-орбиталях не может быть введен непосредственно, поскольку можно говорить только о плотности огибающей волновой функции на ядрах $\mathrm{Si}$ или С. Тем не менее следует учитывать, что, если в политипах $4 H-\mathrm{SiC}$ и $6 H$-SiC плотность неспаренного электрона на $p$-орбиталях С мала и примерно одинакова, на $p$-орбиталях $\mathrm{Si}$ в $4 H-\mathrm{SiC}$ максимальная константа анизотропного суперсверхтонкого (ССТ) взаимодействия $b$ в $4 H-\mathrm{SiC}$, равная $1.26 \mathrm{MHz}$ [46], примерно в 30 раз превышает аналогичную величину для $6 \mathrm{H}-\mathrm{SiC}$.

Важно подчеркнуть, что мы не сравниваем константы СТ-взаимодействия для первых двух координационных сфер, для которых наблюдалась расщепленная структура 
в спектрах ЭПР. Эти величины практически не отражают свойства донорного электрона, которые описываются теорией ЭМ и характерны для зонного электрона. Они определяют глубину уровня, при этом для сравнительно глубоких уровней различие в политипах сглаживается.

Для получения более подробной информации о центре донора $\mathrm{N}$ и волновой функции связанного электрона мы рассмотрим распределение $s$ - и $p$-типа. Используя таблицу Мортона и Престона [56], наблюдаемые СТ-взаимодействия могут быть переведены в спиновую плотность. Результаты были представлены в работе [46]. Установлено, что для $k$-узла в $4 H$-SiC отношение $s: p$ равно 1:4 для плотности на атомах $\mathrm{Si}$ и 2.5:1 для плотности на атомах С. Для узлов $h, k 1$ и $k 2$ в $6 H-\mathrm{SiC}$ отношение $s: p$ равно $1: 1(\mathrm{Si})$ и 19:1 (C); 3.5:1 ( $\mathrm{Si})$ и $6.8: 1(\mathrm{C}) ; 1.6: 1(\mathrm{Si})$ и $2: 1(\mathrm{C})$ соответственно. Таким образом, спиновая плотность, соответствующая наблюдаемым линиям ДЭЯР, является $p$-подобной по своему характеру и локализована в основном на атомах $\mathrm{Si}$ для $k$-узла в $4 H-\mathrm{SiC}$, в то время как для трех узлов в $6 H-\mathrm{SiC}$ спиновая плотность - $s$-подобная по своему характеру и локализована в основном на атомах углерода.

Возможное объяснение различий в волновой функции электронов донора $\mathrm{N}$ в $4 H-\mathrm{SiC}$ и $6 H-\mathrm{SiC}$ можно найти в большом различии в зонной структуре двух политипов и в положении минимумов в зоне Бриллюэна. В результате линейная комбинация атомных орбиталей, описывающих волновую функцию донорного электрона, различна. Таким образом, волновая функция может иметь совершенно иную симметрию и различное распределение $s$ - и $p$-типа на атомах $\mathrm{Si}$ и С. Из расчетов зонной структуры неясно, будут ли лежать зоны $\mathrm{Si}$ или С в минимумах в $4 H-\mathrm{SiC}$ и $6 H$-SiC. Результаты ДЭЯР и ЭПР согласуются с данными в основном в минимумах Si-подобной зоны проводимости в $4 H-\mathrm{SiC}$ (нижняя зона проводимости) и минимумах C-подобной зоны проводимости в $6 \mathrm{H}-\mathrm{SiC}$.

Большая часть спиновой плотности связана с неразрешенным сигналом ДЭЯР вокруг зеемановских частот ядер $\mathrm{Si}$ и $\mathrm{C}$, который состоит из суперпозиции большого числа линий ДЭЯР с очень малым СТ-взаимодействием [46]. В делокализованных центрах этот сигнал, как ожидается, будет очень заметен, в локализованных центрах его практически не будет. Просмотр спектров ДЭЯР на рис. 3, $a$ и в работе [46] показывает, что неразрешенный сигнал действительно очень выражен, с расчетной шириной $1.1 \mathrm{MHz}$. Количество спиновой плотности, включенной в неразрешенный сигнал, можно оценить, используя приближение, что СТ-взаимодействие задается зависимостью $A(\mathbf{r})=A_{0} \exp \left(-\mathbf{r} / \mathbf{r}_{B}\right)$, со спиновой плотностью $A_{0}$ в центре и $\mathbf{r}_{B}-$ радиус Бора волновой функции. Величина спиновой плотности в координационной сфере $\delta \mathbf{r}$ на расстоянии $\mathbf{r}$ от центра дается произведением спиновой плотности $\rho$ при $\mathbf{r}$, вычисленной из $A(\mathbf{r})$, и числом атомов в $\delta \mathbf{r} . \rho \mathbf{r}$ имеет максимум в 2 раза больше радиуса Бора. Используя, например, $\mathbf{r}_{B}=7.2 \AA$ и $A_{0}=4 \mathrm{MHz}, 60 \%$ спиновой плотности, оказывается, возникает от ядер, расположенных между 9 и $30 \AA(A<0.03 \mathrm{mT})$. Представляется разумным предположить, что основная часть спиновой плотности локализуется в кристалле на расстоянии удвоенного радиуса Бора и за его пределами.

В заключение мы сравним свойства доноров азота в $\mathrm{SiC}$ со свойствами аналогичных доноров азота в кристаллах углерода (алмаза) и кремния. Доноры азота впервые были исследованы методом ЭПР в алмазе [57] и кремнии [58,59]. В обоих случаях доноры азота имели глубокие уровни, и их структура резко отличалась от структуры других доноров в кремнии, относящихся к $\mathrm{V}$ группе Периодической таблицы (P, As, Sb), которые характеризуются мелкими уровнями и для описания электронных свойств которых можно было использовать теорию ЭМ. Результаты измерений ЭПР показывают, что центры азота в алмазе и кремнии подвержены сильному тригональному искажению вдоль одной из четырех осей $\langle 111\rangle$, обусловленному нецентральным положением азота. Донорный электрон находится на антисвязывающей орбитали и локализован частично на атоме азота в замещающем положении и частично на одном из ближайших четырех атомов углерода (в алмазе) или кремния (в кремнии). В алмазе неспаренный донорный электрон локализован на 54.9\% на одном углероде (6\% на $s$-орбитали и 94\% на $p$-орбитали) и на $34 \%$ на азоте (19\% на $s$-орбитали и $81 \%$ на р-орбитали), глубина донорного уровня составляет $\sim 2 \mathrm{eV}$. В кремнии неспаренный донорный электрон локализован на $72 \%$ на одном кремнии $(12 \%$ на $s$-орбитали и $88 \%$ на $p$-орбитали) и только на $9 \%$ на азоте (28\% на $s$-орбитали и 72\% на $p$-орбитали), глубина донорного уровня составляет $\sim 0.3 \mathrm{eV}$. Теоретическое рассмотрение образования глубоких донорных уровней азота в кремнии и алмазе было проведено в работах [60-63], где рассматривались эффект Яна-Теллера, псевдоэффект Яна-Теллера и эффект химической связи (chemical rebonding). Поскольку окончательно вопрос об образовании глубоких донорных уровней азота в кремнии и алмазе не решен, то и сравнительный анализ различного поведения азота в кремнии и алмазе, с одной стороны, и в $\mathrm{SiC}-$ с другой, насколько нам известно, не проводился.

Для доноров азота в $\mathrm{SiC}$ наблюдается совершенно иная ситуация, которая даже качественно не может быть сопоставлена с поведением глубоких доноров азота в кремнии и алмазе. В $\mathrm{SiC}$ азот образует доноры со сравнительно мелкими уровнями и, главное, не возникает связи между азотом и ближайшим атомом решетки и не возникает существенного понижения симметрии, как это происходит для кристаллов кремния и алмаза. Если в случае гексагональных политипов $\mathrm{SiC}$ можно предположить, что определенную роль играет аксиальная симметрия кристалла ( $g$-фактор и константа СТ-структуры имеют слабую аксиальную симметрию вдоль оси с кристалла), а также возможно частичное проявление нецентрального положения азота в $k$-позициях, то для кубической модификации $\mathrm{SiC}(3 C-\mathrm{SiC})$ имеет место 
такая же симметрия, как для алмаза или кремния. Таким образом, представляется весьма перспективным построение общей теории, объясняющей принципиальное различие в поведении донорных примесей азота в кристаллах кремния и алмаза, с одной стороны, и $\mathrm{SiC}-$ с другой.

Сильная делокализация неспаренного электрона донора азота в решетке карбида кремния должна учитываться при создании структур на основе спиновых центров в этом материале, работающих при комнатной температуре. Для многих применений необходимо исключить, или по крайней мере минимизировать влияние спиновой системы доноров азота на когерентные свойства спиновых центров. Результаты настоящей работы и предыдущих исследований, в которой однозначно установлен различный характер распределения спиновой плотности в политипах $4 H-\mathrm{SiC}$ и $6 H-\mathrm{SiC}$, а также электронная структура доноров азота должны помочь решить эти проблемы. Установлено, что спиновая плотность донорного электрона азота в $4 H$-SiC распространяется в основном по атомам кремния, тогда как в $6 \mathrm{H}$-SiC - по атомам углерода. Спиновые центры, в которых обнаружено оптически индуцированное выстраивание спиновых уровней, в своей структуре имеют парамагнитную вакансию кремния. Так как ближайшими к вакансии кремния являются атомы углерода, можно предположить, что именно эти атомы будут вносить основной вклад в спиновую релаксацию неспаренных электронов вакансии кремния. Таким образом, спиновые центры в политипе $4 H$-SiC могут быть более устойчивы к взаимодействиям с неспаренными донорными электронами, поскольку в этом случае электроны не локализуются на ближайшей к спиновому центру координационной сфере.

\section{Список литературы}

[1] A. Gruber, A. Dräbenstedt, C. Tietz, L. Fleury, J. Wrachtrup, C. von Borczyskowski. Science 276, 2012 (1997).

[2] F. Jelezko, I. Popa, A. Gruber, C. Tietz, J. Wrachtrup, A. Nizovtsev, S. Kilin. Appl. Phys. Lett. 81, 2160 (2002).

[3] F. Jelezko, T. Gaebel, I. Popa, A. Gruber, J. Wrachtrup. Phys. Rev. Lett. 92, 076401 (2004).

[4] F. Jelezko, J. Wrachtrup. Phys. Status Solidi A 203, 3207 (2006).

[5] D.D. Awschalom, R. Epstein, R. Hanson. Sci. Am. 84-91 (2007).

[6] D. Di Vincenzo. Nature Mater. 9, 468 (2010).

[7] P.G. Baranov, I.V. Il'in, E.N. Mokhov, M.V. Muzafarova, S.B. Orlinskii, J. Schmidt. JETP Lett. 82, 441 (2005).

[8] P.G. Baranov, A.P. Bundakova, I.V. Borovykh, S.B. Orlinskii, R. Zondervan, J. Schmidt. JETP Lett. 86, 202 (2007).

[9] J.R. Weber, W.F. Koehl, J.B. Varley, A. Janotti, B.B. Buckley, C.G. Van de Walle, D.D. Awschalom. Proc. Nat. Acad. Sci. USA 107, 8513 (2010).

[10] P.G. Baranov, A.P. Bundakova, A.A. Soltamova, S.B. Orlinskii, I.V. Borovykh, R. Zondervan, R. Verberk, J. Schmidt. Phys. Rev. B 83, 125203 (2011).

[11] A.G. Smart, Phys. Today 65, 10 (2012).
[12] W.F. Koehl, B.B. Buckley, F.J. Heremans, G. Calusine, D.D. Awschalom. Nature 479, 84 (2011).

[13] V.A. Soltamov, A.A. Soltamova, P.G. Baranov, I.I. Proskuryakov. Phys. Rev. Lett. 108, 226402 (2012).

[14] D. Riedel, F. Fuchs, H. Kraus, S. Vath, A. Sperlich, V. Dyakonov, A.A. Soltamova, P.G. Baranov, V.A. Ilyin, G.V. Astakhov. Phys. Rev. Lett. 109, 226402 (2012).

[15] F. Fuchs, V.A. Soltamov, S. Vath, P.G. Baranov, E.N. Mokhov, G.V. Astakhov, V. Dyakonov. Sci. Rep. 3, 1637 (2013).

[16] S. Castelletto, B. C. Johnson, A. Boretti. Adv. Opt. Mater. 1, 609 (2013).

[17] A.L. Falk, B.B. Buckley, G. Calusine, W.F. Koehl, V.V. Dobrovitski, A. Politi, C.A. Zorman, P.X.L. Feng, D.D. Awschalom. Nature Commun. 4, 1819 (2013).

[18] T.C. Hain, F. Fuchs, V.A. Soltamov, P.G. Baranov, G.V. Astakhov, T. Hertel, V. Dyakonov. J. Appl. Phys. 115, 133508 (2014).

[19] A. Muzha, F. Fuchs, N.V. Tarakina, D. Simin, M. Trupke, V.A. Soltamov, E.N. Mokhov, P.G. Baranov, V. Dyakonov, A. Krueger, G.V. Astakhov. Appl. Phys. Lett. 105, 243112 (2014).

[20] H. Kraus, V.A. Soltamov, D. Riedel, S. Vath, F. Fuchs, A. Sperlich, P.G. Baranov, V. Dyakonov, G.V. Astakhov. Nature Phys. 10, 157 (2014).

[21] P.V. Klimov, A.L. Falk, B.B. Buckley, D.D. Awschalom. Phys. Rev. Lett. 112, 087601 (2014).

[22] A.L. Falk, P.V. Klimov, B.B. Buckley, V. Ivady, I.A. Abrikosov, G. Calusine, W.F. Koehl, A. Gali, D.D. Awschalom. Phys. Rev. Lett. 112, 187601 (2014).

[23] H. Kraus, V.A. Soltamov, F. Fuchs, D. Simin, A. Sperlich, P.G. Baranov, G.V. Astakhov, V. Dyakonov. Sci. Rep. 4, 5303 (2014).

[24] V.A. Soltamov, B.V. Yavkin, D.O. Tolmachev, R.A. Babunts, A.G. Badalyan, V.Yu. Davydov, E.N. Mokhov, I.I. Proskuryakov, S.B. Orlinskii, P.G. Baranov. Phys. Rev. Lett. 115, 247602 (2015).

[25] A.L. Falk, P.V. Klimov, V. Ivady, K. Szasz, D.J. Christle, W.F. Koehl, A. Gali, D.D. Awschalom. Phys. Rev. Lett. 114, 247603 (2015).

[26] S.G. Carter, O.O. Soykal, P. Dev, S.E. Economou, E.R. Glaser. Phys. Rev. B 92, 161202 (2015).

[27] D. Simin, F. Fuchs, H. Kraus, A. Sperlich, P.G. Baranov, G.V. Astakhov, V. Dyakonov. Phys. Rev. Appl. 4, 014009 (2015).

[28] S.-Yu. Lee, M. Niethammer, J. Wrachtrup. Phys. Rev. B 92, 115201 (2015).

[29] D.J. Christle, A.L. Falk, P. Andrich, P.V. Klimov, J. Hassan, N.T. Son, E. Janzen, T. Ohshima, D.D. Awschalom. Nature Mater. 14, 160 (2015).

[30] M. Widmann, S.-Yu. Lee, T. Rendler, N.T. Son, H. Fedder, S. Paik, Li-Ping Yang, N. Zhao, S. Yang, I. Booker, A. Denisenko, M. Jamali, S. Ali Momenzadeh, I. Gerhardt, T. Ohshima, A. Gali, E. Janzen, J. Wrachtrup. Nature Mater. 14, 164 (2015).

[31] В.С. Вайнер, В.А. Ильин. ФТТ 23, 3659 (1981).

[32] G. Feher. Phys. Rev. 114, 1219 (1959).

[33] R.C. Fletcher, W.A. Yager, G.L. Pearson, F.R. Merritt. Phys. Rev. 95, 844 (1954).

[34] J.L. Ivey R.L. Mieher. Phys. Rev. B 11, 849 (1975).

[35] A.V. Duijn-Arnold, J. Mol, R. Verberk, J. Schmidt, E.N. Mokhov, P.G. Baranov. Phys. Rev. B 60, 15829 (1999). 
[36] W.J. Choyke, L. Patrick. Phys. Rev. 127, 1868-1877 (1962); W.J. Choyke, D.R. Hamilton, L. Patrick. Phys. Rev. 133, A1163 (1964).

[37] P.J. Colwell, M.V. Klein. Phys. Rev. B 6, 498 (1972).

[38] H.H. Woodbury, G.W. Ludwig. Phys. Rev. 124, 1083 (1961); G.W. Ludwig, H.H. Woodbury. Solid State Phys. 13, 223 (1962).

[39] G.E.G. Hardeman, G.B. Gerritsen. Phys. Rev. Lett. 20, 623 (1966).

[40] L. Patrick. Phys. Rev. B 5, 2198 (1972); L. Patrick. Phys. Rev. 138, A1477 (1965).

[41] E.N. Kalabukhova, N.N. Kabdin, S.N. Lukin. Sov. Phys. Solid State 29, 8 (1987).

[42] S. Greulich-Weber, M. Feege, J.-M. Spaeth, E.N. Kalabukhova, S.N. Lukin, E.N. Mokhov. Solid State Commun. 93, 393 (1995).

[43] S. Greulich-Weber. Phys. Status Solidi A 162, 95 (1997).

[44] W. Kohn, J.M. Luttinger. Phys. Rev. 97, 1721 (1955).

[45] W. Kohn. In: Solid State Phys. / Ed. by F. Seitz D. Turnbull. Academic Press Inc., N. Y. (1957). V. 5. 257-320.

[46] A.V. Duijn-Arnold, R. Zondervan, J. Schmidt, P.G. Baranov, E.N. Mokhov. Phys. Rev. B 64, 085206 (2001).

[47] K. Szász, X.T. Trinh, N.T. Son, E. Janzén, A. Gali. J. Appl. Phys. 115, 073705 (2014).

[48] N.T. Son, E. Janzen, J. Isoya, S. Yamasaki. Phys. Rev. B 70, 193207 (2004)

[49] D.V. Savchenko, E.N. Kalabukhova, V.S. Kiselev, J. Hoentsch, A. Poppl. Phys. Status Solidi B 246, 1908 (2009).

[50] D.V. Savchenko, E.N. Kalabukhova, A. Poppl, B.D. Shanina. Phys. Status Solidi B 249, 2167 (2012).

[51] P.G. Baranov, B.Ya. Ber, O.N. Godisov, I.V. Il'in, A.N. Ionov, E.N. Mokhov, M.V. Muzafarova, A.K. Kaliteevskii, M.A. Kaliteevskii, P.S. Kop'ev. Phys. Solid State 47, 2219 (2005); ФTT 47, 12, 2127 (2005).

[52] N.T. Son, J. Isoya, T. Umeda, I.G. Ivanov, A. Henry, T. Ohshima, E. Janzen. Appl. Magn. Res. 39, 49 (2010).

[53] J.-M. Spaeth, J.R. Niklas, R.H. Bartram. Structural Analysis of Point Defect in Solids. Springer Ser. Solid State Sci. 43. Springer-Verlag, Berlin, Heidelberg, N. Y. (1992).

[54] E.N. Kalabukhova, S.N. Lukin, W.C. Mitchel. Mater. Sci. Forum 433-436, 499 (2003).

[55] N.T. Son, J. Isoya, S. Yamasaki, E. Janzen. In: Book of Abstracts of the 5th Eur. Conf. Silicon Carbide Rel. Mater., Bolonga, Italy (2004). (CNR-IMM, Area Della Ricerca, Bologna, 2004).

[56] J.R. Morton, K.F. Preston. J. Magn. Res. 30, 377 (1978).

[57] W.V. Smith, P.P. Sorokin, I.L. Gelles, G.J. Lasher. Phys. Rev. 115, 1546 (1959).

[58] K.L. Brower. Phys. Rev. Lett. 44, 1627 (1980).

[59] K. Murakami, H. Kuribayashi, K. Masuda. Phys. Rev. B 38, 1589 (1988).

[60] R.P. Messmer, G.D. Watkins. Phys. Rev. B 7, 2568 (1973).

[61] G.G. DeLeo, W.B. Fowler, G.D. Watkins. Phys. Rev. B 29, 3193 (1984).

[62] S.T. Pantelides, W.A. Harrison, F. Yndurain. Phys. Rev. B 34, 6038 (1986).

[63] F.G. Anderson. Phys. Rev. B 39, 5392 (1989). 